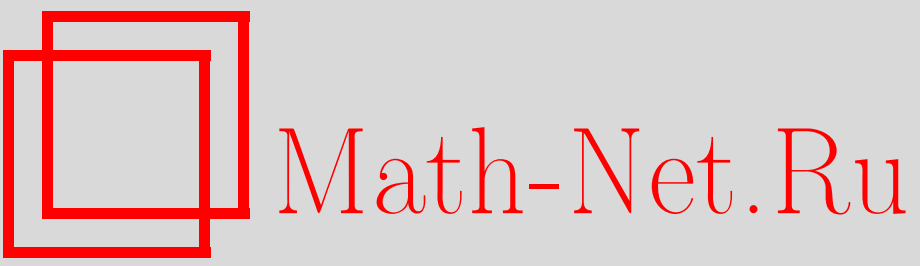

В. В. Арестов, Приближение неограниченных операторов ограниченными и родственные экстремальные задачи, УМН, 1996, том 51, выпуск 6, 89-124

DOI: https://doi.org/10.4213/rm1019

Использование Общероссийского математического портала Math-Net.Ru подразумевает, что вы прочитали и согласны с пользовательским соглашением

http://www.mathnet.ru/rus/agreement

Параметры загрузки:

IP: 3.95 .254 .165

26 апреля 2023 г., 18:04:21 


\title{
ПРИБЛИЖЕНИЕ НЕОГРАНИЧЕННЫХ ОПЕРАТОРОВ ОГРАНИЧЕННЫМИ И РОДСТВЕННЫЕ ЭКСТРЕМАЛЬНЫЕ ЗАДАЧИ
}

\author{
B. В. Арестов
}

СОДЕРЖАНИЕ

$\S 0$. Введение ..................................................... 89

$\S 1$. Оценка Стечкина величины наилучшего приближения оператора ... 91

$\S 2$. Связь задачи Стечкина с некорректной задачей восстановления значений неограниченного оператора ......................... 92

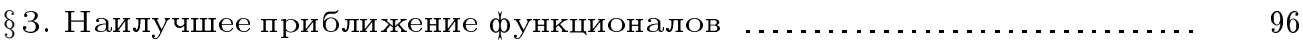

$\S 4$. Наилучшее приближение операторов дифференцирования и неравенства между нормами производных для функций одного переменного на оси и полуоси

$\S 5$. Приближение операторов дифференцирования на классах функций многих переменных ....................................... 105

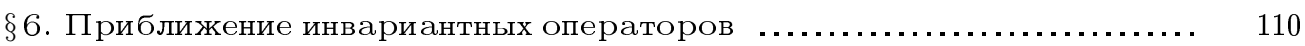

$\S 7$. Приближение одного класса дифференцируемых функций другим классом более гладких функций …............................ 117

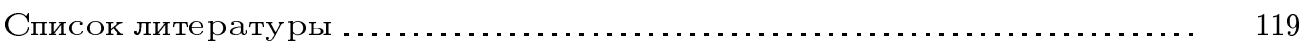

\section{$\S$ 0. Введение}

Задача о наилучшем приближении неограниченного линейного оператора линейными ограниченными операторами на классе элементов банахова пространства появилась в исследованиях С.Б. Стечкина в 1965 году [1]. В его работе [2] 1967 года была дана постановка задачи, приведены первые принципиальные результаты и дано решение задачи для операторов дифференщирования малого порядка.

Точная постановка задачи такова. Пусть $X, Y$ - банаховы пространства; $A: X \rightarrow Y$ - некоторьй оператор (необязательно линейный) с областью определения $D(A) \subset X$; $Q$ - некоторый класс элементов из $D(A) ; \mathscr{L}(N)=\mathscr{L}(N ; X, Y)$ - множество линейных

Работа выполнена при финансовой поддержке фонда ИНТАС (Международная ассоциация содействия сотрудничеству с учеными независимых государств бывшего Советского Союза), грант INTAS 94-4070. 
ограниченных операторов из $X$ в $Y$, норма которых $\|T\|=\|T\|_{X \rightarrow Y}$ не превосходит числа $N \geqslant 0$. Величина

$$
U(T)=\sup \left\{\|A x-T x\|_{Y}: x \in Q\right\}
$$

является уклонением оператора $T \in \mathscr{L}(N)$ от оператора $A$ на классе $Q$, а

$$
E(N)=E(N ; A, Q)=\inf \{U(T): T \in \mathscr{L}(N)\}
$$

есть наилучшее приближение оператора $A$ множеством ограниченных операторов $\mathscr{L}(N)$ на классе $Q$. Задача состоит в вычислении (исследовании) величины $E(N)$, нахож дении (исследовании вопроса существования, единственности, характеризации) экстремального оператора, на котором в (0.2) достигается нижняя грань. Эта задача в дальнейшем будет назьваться задачей Стечкина или задачей (0.2).

Изучение (а, вероятно, и возникновение) этой задачи происходило в тесной взаимосвязи с исследованием экстремальных задач теории приближения функций, теории некорректных задач, вычислительной математики. Особенно большое взаимное влияние оказали задача Стечкина (в особенности, задача о наилучшем приближении операторов дифференцирования ограниченными операторами в функциональных пространствах на числовой оси и полуоси) с одной стороны, а с другой - точные неравенства меж ду нормами производных дифференцируемых функций (неравенства Колмогорова) и некорректная задача оптимальной равномерной регуляризации вычисления значений неограниченного оператора на элементах, заданных с ошибкой.

K настоящему времени в задаче Стечкина получены следующие результаты. Выяснена взаимосвязь этой задачи с другими экстремальными задачами. Установлены количественные соотношения между модулем непрерьвности линейного неограниченного оператора на классе элементов пространства, наилучшим приближением такого оператора линейными ограниченными операторами и некорректной задачей оптимального равномерного восстановления значений оператора на элементах класса в предположении, что эти элементы заданы неточно (с известной погрешностью). Получены двойственные соотношения между первыми двумя задачами и, соответственно, наилучшим и наилучшим линейньм приближениями одного класса элементов другим. Получен ряд общих теорем существования и характеризации экстремального приближаюшего оператора. Обстоятельно изучено наилучшее приближение функционалов. Подробно исследовано приближение операторов, инвариантных относительно некоторых полугрупп (групп) преобразований. Более полно и результативно изучено наилучшее приближение операторов, инвариантных относительно сдвига, в пространствах $L_{\gamma}\left(\mathbb{R}^{m}\right), 1 \leqslant \gamma \leqslant \infty$. Дано решение задачи для конкретных операторов в классических функциональных пространствах. При этом наиболее полно исследовано наилучшее приближение операторов дифференцирования порядка $k$ на классе $n$ раз дифференцируемых функций $(0 \leqslant k<n)$ в пространствах $L_{\gamma}=L_{\gamma}(S)$ на числовой оси $S=(-\infty, \infty)$ и полуоси $S=[0, \infty)$. На этом пути найдены наилучшие константы в неравенствах Колмогорова в ряде новых случаев. Вычислено наилучшее приближение операторов дифференцирования в частных производных на классах функций многих переменных в ряде случаев. 
Цель данной работы автор видел в том, чтобы изложить результаты С. Б. Стечкина, относящиеся к задаче $(0.2)$, их дальнейшее развитие и современное состояние исследований по этой тематике; при этом основное внимание уделено точным результатам. Большой обзорньй материал по этой проблематике имеется в [3]-[6]; данная работа и недавно вьшедшая обзорная статья [7] автора и В. Н. Габушина пересекаются и сушественно дополняют друг друга.

\section{$\S 1$. Оценка Стечкина величины наилучшего приближения оператора}

Для оператора $A$, класса $Q$, пространств $X$ и $Y$ каждую из двух функций

$$
\begin{aligned}
& \Omega(\delta)=\sup \left\{\left\|A x_{1}-A x_{2}\right\|_{Y}: x_{1}, x_{2} \in Q,\left\|x_{1}-x_{2}\right\|_{X} \leqslant \delta\right\} \\
& \omega(\delta)=\sup \left\{\|A x\|_{Y}: x \in Q,\|x\|_{X} \leqslant \delta\right\}
\end{aligned}
$$

вешественного переменного $\delta \in[0, \infty)$ назьвают модулем непрерывности оператора $A$ на классе $Q$. Если оператор $A$ линейный и множество $Q$ центрально симметричное и вьпуклое, то эти характеристики связаны соотношением [8], [9]

$$
\Omega(2 \delta)=2 \omega(\delta), \quad \delta \geqslant 0
$$

Следуюшая теорема С. Б. Стечкина [2] дает простую, но часто используемую и эффективную оценку снизу величины наилучшего приближения (0.2) оператора через его модуль непрерьвности. В этой теореме и ниже используются обозначения

$$
\begin{aligned}
& \Delta(N)=\Delta(N, Q)=\sup \{\omega(\delta)-N \delta: \delta \geqslant 0\} \\
&=\sup \left\{\|A x\|_{Y}-N\|x\|_{X}: x \in Q\right\}, \\
& \ell(\delta)=\inf \{E(N)+N \delta: N \geqslant 0\} .
\end{aligned}
$$

Tеорема 1.1. Если $A$ - однородный (в частности, линейный) оператор, $Q$ центрально симметричное выпуклое множество из области определения оператора А, то имеют место неравенства

$$
\begin{gathered}
E(N) \geqslant \Delta(N), \quad N \geqslant 0, \\
\omega(\delta) \leqslant \ell(\delta), \quad \delta \geqslant 0 .
\end{gathered}
$$

Если при этом существует әлемент $x \in Q$ и линейный ограниченный оператор Т со свойствами

$$
\|A x\|=U(T)+\|T\|\|x\|,
$$

то имеют место равенства

$$
\omega(\|x\|)=\|A x\|, \quad E(\|T\|)=U(T)=\|A x\|-\|T\|\|x\|,
$$


u, следовательно, оператор $T$ является зкстремальным в задаче (0.2) при $N=\|T\|$, а әлемент $x-$ в задаче (1.2) при $\delta=\|x\|$.

Для любого элемента $x \in Q$ и линейного ограниченного оператора вьполняется неравенство $U(T) \geqslant\|A x\|-\|T\|\|x\|$. Поэтому, если удалось подобрать (угадать) элемент $x \in Q$ и оператор $T$ так, чтобы выполнялось неравенство $U(T) \leqslant\|A x\|-\|T\|\|x\|$, то согласно предыдушей теореме мы получаем решение обеих задач $(0.2)$ и (1.2), соответственно, при $N=\|T\|$ и $\delta=\|x\|$. Именно эта схема Стечкина применялась в большинстве работ, содержаших точное решение задачи (0.2).

Вопрос о том, при каких условиях неравенства (1.6), (1.7) обрашаются в равенства, является важным и до сих пор нерешенным. Известно [10], что для функционалов оба эти неравенства обрашаются в равенство (см. теоремы 3.1 и 3.2 ниже). В обшем случае равенства в (1.6), (1.7) не имеют места. Может случиться, что при любом $\delta>0$ величина $\omega(\delta)$ конечна, а величина $E(N)$ не является конечной для всех $N>0$. Возможно также, что все рассматриваемые величины конечны, но неравенства (1.6), (1.7) являются строгими. Эта ситуация имеет место уже для приближения операторов дифференцирования в пространствах $L_{p}$ и будет обсуждаться ниже.

\section{$\S$ 2. Связь задачи Стечкина с некорректной задачей восстановления значений неограниченного оператора}

1. Некоторые задачи вычислительной математики, теории функций, других разделов математики являются некорректными задачами восстановления значений оператора $A$ на элементах класса $Q$, лежашего в области определения $D(A)$ оператора $A$, в предположении, что элементы класса $Q$ заданы с известной погрешностью; восстановление осушествляется с помошью некоторого множества $\mathscr{R}$ операторов (однозначных отображений) пространства $X$ в $Y$. В качестве $\mathscr{R}$, как правило, берется одно из следующих множеств отображений: множество $\mathscr{O}=\mathscr{O}(X, Y)$ всех отображений пространства $X$ в пространство $Y$, множество $\mathscr{L}=\mathscr{L}(X, Y)$ линейных операторов из $X$ в $Y$, множество $\mathscr{B}=\mathscr{B}(X, Y)$ линейных ограниченных операторов из $X$ в $Y$, а также ранее определенное множество $\mathscr{L}(N)=\mathscr{L}_{N}=\mathscr{L}(N ; X, Y)$ линейных ограниченных операторов из $X$ в $Y$, норма которых не превосходит числа $N \geqslant 0$. В дальнейшем через $\delta$ будет обозначаться неотрицательньй параметр, характеризуюший погрешность в задании элементов класса $Q$. Для числа $\delta \geqslant 0$ и оператора $T \in \mathscr{R}$ полагаем

$$
U_{\delta}(T)=U_{\delta}(T ; A, Q)=\sup \left\{\|A x-T \eta\|_{Y}: x \in Q, \eta \in X,\|x-\eta\|_{X} \leqslant \delta\right\} .
$$

Тогда

$$
\mathscr{E}_{\delta}(\mathscr{R})=\mathscr{E}_{\delta}(\mathscr{R} ; A, Q)=\inf \left\{U_{\delta}(T): T \in \mathscr{R}\right\}
$$

есть величина наилучшего восстановления (наименьшая погрешность восстановления) оператора $A$ с помошью множества отображений (методов восстановления) $\mathscr{R}$ на элементах класса $Q$, заданных с известной погрешностью $\delta$. Построению и изучению конкретных методов восстановления, в частности, оценкам величины (2.1) для конкретных методов и исследованию задачи (2.2) (а также аналогичным и более 
обшим задачам) посвящено большое число работ А.Н. Тихонова, М. М. Лаврентьева, В.К. Иванова, В.Н. Страхова, С. Б. Стечкина, Ю.Н. Субботина, В.Н. Габушина, В. Г. Романова, В. А. Морозова, В. В. Васина, В.П. Тананы, В.Я. Арсенина, В. В. Иванова, В. А. Винокурова, А. И. Гребенникова, А. Г. Марчука, К. Ю. Осипенко, Ш. Мичелли, Т. Ривлина, Дж. Трауба, Х. Вожняковского, Б. Боянова, Г. Г. Магарил-Ильяева, А. А. Женсыкбаева и многих других (см. монографии [11]-[20], работы [21]-[31] и приведенную там библиографию).

Для оценки величин (2.1) и (2.2) используется модуль непрерьвности (1.1), (1.2) оператора $A$ на классе $Q$. Так, в работе [8] показано, что если оператор $A$ линейный и множество $Q$ центрально симметричное, то для метода квазирешений $T_{K}$ В. К. Иванова справедливы оценки $\omega(\delta) \leqslant U_{\delta}\left(T_{K}\right) \leqslant 2 \omega(\delta)$; подобные оценки справедливы и для многих других методов регуляризации (см. монографии [11]-[20], обзор [28] и приведенную в них библиографию). Известно (см. работы [22], [32], [26] и теорему 2.1 ниже), что если оператор $A$ однородный, то для любого метода $T \in \mathscr{O}$ имеет место оценка снизу $U_{\delta}(T) \geqslant \omega(\delta)$. Следовательно,

$$
\omega(\delta) \leqslant \mathscr{E}_{\delta}(\mathscr{O}) \leqslant 2 \omega(\delta) .
$$

Следуюшее утверждение (теорема 2.1) в той или иной степени обшности приводилось, начиная с 1965 года, в ряде сообщений С.Б. Стечкина на научных семинарах и конференциях. В приводимой здесь форме оно содержалось, в частности, в его докладе на Международной конференции по теории функций в г. Будапеште в 1969 году (см. работу [33]); впервые оно было опубликовано в [3]. Это утверждение показывает, что не только задачи $(2.2),(1.2)$, но и задача $(0.2)$ между собой взаимосвязаны. Близкий результат имеется в [27]. Решение задачи Стечкина (0.2) впервые явно использовалось в работе [34] для получения порядков величины $\mathscr{E}_{\delta}(\mathscr{L})$ для операторов дифференцирования.

Теорема 2.1. Если $A$-однородный оператор, $Q$ - чентрально симметричное выпуклое множество, то при любом $\delta>0$

$$
\omega(\delta) \leqslant \mathscr{E}_{\delta}(\mathscr{O}) \leqslant \mathscr{E}_{\delta}(\mathscr{B})=\mathscr{E}_{\delta}(\mathscr{L}) \leqslant \ell(\delta) .
$$

Если при этом существуют әлемент $x \in Q$ и оператор $T \in \mathscr{B}$ со свойством (1.8), то имеют место равенства

$$
\|A x\|=\omega(\delta)=\mathscr{E}_{\delta}(\mathscr{O})=\mathscr{E}_{\delta}(\mathscr{L}), \quad \delta=\|x\|
$$

2. Соотношения (2.4) можно дополнить и уточнить. Для ограниченного подмножества $M$ нормированного пространства $Y$ числа

$$
\begin{gathered}
d(M)=d(M)_{Y}=\sup \left\{\left\|y_{1}-y_{2}\right\|_{Y}: y_{1}, y_{2} \in M\right\} \\
r(M)=r(M)_{Y}=\inf _{z \in Y} \sup _{y \in M}\|z-y\|_{Y}
\end{gathered}
$$


назьвают, соответственно, диаметром и чебъиевским радиусом множества $M$; если существует элемент $z=z(M) \in Y$, на котором в (2.6) достигается нижняя грань, то этот элемент называется чебышевским центром $M$. Величину

$$
H(Y)=\sup \left\{\frac{r(M)}{d(M)}: M \subset Y\right\}
$$

назьвают константой (или модулем) Юнга пространства $Y$ [35]. Отметим некоторые известные факты относительно этой величины. Очевидно, всегда $1 / 2 \leqslant H(Y) \leqslant 1$. Г. Юнг доказал, что

$$
H\left(\mathbb{R}^{m}\right)=\left\{\frac{m}{2(m+1)}\right\}^{1 / 2} .
$$

Известно (см., например, [35]), что для бесконечномерного гильбертова пространства $H(Y)=1 / \sqrt{2}$. С. А. Пичугов показал [36], что для пространств $L_{p}, 1 \leqslant p<\infty$,

$$
H\left(L_{p}\right)=2^{-1 / \theta}, \quad \theta=\max \left(p, p^{\prime}\right), \quad 1 / p+1 / p^{\prime}=1 .
$$

Для элемента $\eta \in X$ введем множество $Q(\eta, \delta)=Q \cap V(\eta, \delta)$, где $V(\eta, \delta)=\{z \in X$ : $\|\eta-z\| \leqslant \delta\}-$ (замкнутьй) шар радиуса $\delta$ с центром в точке $\eta$ пространства $X$. Пусть, далее, $Q(\delta)=\bigcup_{x \in Q} V(x, \delta)$ есть $\delta$-расширение множества $Q$.

Теорема 2.2. При любом $\delta>0$ имеют место равенство

$$
\mathscr{E}_{\delta}(\mathscr{O})=\sup \{r(A Q(\eta, \delta)): \eta \in Q(\delta)\}
$$

и неравенства

$$
\frac{1}{2} \Omega(2 \delta) \leqslant \mathscr{E}_{\delta}(\mathscr{O}) \leqslant H(Y) \Omega(2 \delta) .
$$

Утверждения, близкие приведенным в этой теореме, можно найти во многих работах (см. [23], [25], [3], [27], [28], [4], [5] и приведенную там библиографию); в сформулированном здесь виде оно содержится в [5]. Равенство (2.9) означает, что в задаче (2.2) всегда наилучшим (в обшем случае, конечно, нелинейным) является метод восстановления, который элементу $\eta \in Q(\delta)$ сопоставляет чебышевский центр множества $A Q(\eta, \delta)$ (если таковой существует); по-видимому, впервые чебышевские центры в задачах теории приближения использовал Голомб [37].

Если оператор $A$ линейньй, а множество $Q$ центрально симметричное и вьпуклое, то в силу (1.3) соотношение (2.10) принимает вид

$$
\omega(\delta) \leqslant \mathscr{E}_{\delta}(\mathscr{O}) \leqslant 2 H(Y) \omega(\delta)
$$

здесь второе неравенство точнее соответствуюшего неравенства в $(2.3)$.

3. Последнее неравенство в (2.4) дает оценку сверху погрешности $\mathscr{E}_{\delta}(\mathscr{L})$ линейного восстановления оператора $A$ через величину $\ell(\delta)$ (и, значит, через $E(N)$ ). В свою 
очередь, $\ell(\delta)$ и $E(N)$ можно оценить сверху через $\mathscr{E}_{\delta}(\mathscr{L})$. Приводимая ниже оценка из [5] основана на более ранних оценках из [3], [4]. Введем функцию

$(2.12) \Phi(\alpha, \beta)=\inf \left\{\max \left(\|a+b\|_{Y},\|a-b\|_{Y}\right):\|a\|_{Y}=\alpha,\|b\|_{Y}=\beta\right\}, \quad \alpha>0, \beta>0$.

Нетрудно проверить, что при любых $\alpha>0, \beta>0$ для этой функции справедливы оценки

$$
\max (\alpha, \beta) \leqslant \Phi(\alpha, \beta) \leqslant \alpha+\beta .
$$

Функция $\Phi$ выражается через модуль выпуклости единичной сферы пространства $Y$ [38, лемма 1.4]. Используя этот факт и результат Кларксона-Ханнера (см., например, [38]), получаем, что если $Y$ есть пространство $L_{p}$, то

$$
\begin{aligned}
& \Phi(\alpha, \beta)=\left(\alpha^{p}+\beta^{p}\right)^{1 / p}, \quad 2 \leqslant p \leqslant \infty, \\
& \Phi(\alpha, \beta)=\left(\frac{(\alpha+\beta)^{p}+|\alpha-\beta|^{p}}{2}\right)^{1 / p}, \quad 1 \leqslant p \leqslant 2 .
\end{aligned}
$$

В частности, если $Y$ есть $L_{1}$ или $L_{\infty}$, то

$$
\Phi(\alpha, \beta)=\max (\alpha, \beta) .
$$

Для гильбертова пространства

$$
\Phi(\alpha, \beta)=\left(\alpha^{2}+\beta^{2}\right)^{1 / 2} .
$$

С помощью функции $\Phi$ определим величину

$$
\ell^{*}(\delta)=\inf \{\Phi(E(N), N \delta): N>0\} .
$$

Для этой величины, очевидно, имеем

$$
\ell^{*}(\delta) \geqslant \inf \{\max (E(N), N \delta): N>0\} \geqslant \frac{1}{2} \ell(\delta) .
$$

TEOPEMA 2.3. При любом $\delta>0$

$$
\ell^{*}(\delta) \leqslant \mathscr{E}_{\delta}(\mathscr{L}) .
$$

В силу $(2.4)$ и $(2.17)$ величины $\ell(\delta)$ и $\mathscr{E}_{\delta}(\mathscr{L})$ связаны неравенствами

$$
\frac{1}{2} \ell(\delta) \leqslant \mathscr{E}_{\delta}(\mathscr{L}) \leqslant \ell(\delta) .
$$

4. Более обшей, чем (2.2) (и (0.2)), является задача восстановления значений оператора $A$ на элементах $x \in Q$ по точному или приближенному значению $I x$ некоторого информационного оператора $I$ на элементе $x$. Она часто возникает в различных разделах математики, хорошо изучена (см., например, [25], [28], [39], [5], [30], [31] и приведенную там библиографию) и состоит в следуюшем. Пусть $X$ - некоторое множество, 
$Y$ и $Z$ - метрические пространства, $A$ - оператор из $X$ в $Y$ с областью определения $D(A) \subset X, Q$ - подмножество множества $D(A), I$ - отображение $Q$ в $Z, \mathscr{R}$ - семейство (однозначных) отображений пространства $Z$ в $Y$. Для числа $\delta \geqslant 0$ и оператора $T \in \mathscr{R}$

$$
U_{\delta}(T)=U_{\delta}(T ; A, Q, I)=\sup \left\{\|A x-T \eta\|_{Y}: x \in Q, \eta \in Z,\|I x-\eta\|_{Z} \leqslant \delta\right\}
$$

есть погрешность восстановления методом $T$ оператора $A$ на элементах класса $Q$, заданных точно (если $\delta=0$ ) или приближенно (если $\delta>0$ ) информационным оператором $I$. Тогда

$$
\mathscr{E}_{\delta}^{*}(\mathscr{R})=\mathscr{E}_{\delta}^{*}(\mathscr{R} ; A, Q, I)=\inf \left\{U_{\delta}(T ; A, Q, I): T \in \mathscr{R}\right\}
$$

есть величина наилучшего восстановления (с помошью семейства $\mathscr{R}$ ). В теории квадратурных формул и других разделах математики возникают более общие задачи. Именно, пусть $\mathscr{I}$ есть некоторое семейство информационных операторов $I$ из $X$ в $Y$ и при каждом $I \in \mathscr{I}$ семейство $\mathscr{R}=\mathscr{R}_{I}$ зависит, вообше говоря, от $I$. Задача состоит в исследовании величины

$$
\inf \left\{\mathscr{E}_{\delta}^{*}\left(\mathscr{R}_{I} ; A, Q, I\right): I \in \mathscr{I}\right\} .
$$

Нетрудно понять, что задачи (0.2), (2.2), (2.20), (2.21) вкладьваются в следующую общую и естественную схему. Пусть $W$ - подмножество декартова произведения $X \times Y$ пространств $X$ и $Y, \mathscr{Q}=\operatorname{pr}_{X} W$ - проекция множества $W$ на $X, \mathscr{R}$ - некоторое семейство отображений $\mathscr{Q}$ в $Y$. Задача состоит в исследовании величины

$$
\mathscr{E}(\mathscr{R}, W)=\inf \{U(T): T \in \mathscr{R}\}
$$

где

$$
U(T)=U(T, W)=\sup \left\{\|y-T x\|_{Y}:(x, y) \in W\right\}
$$

Величину $\mathscr{E}(\mathscr{R}, W)$ можно интерпретировать как наилучшее приближение на $\mathscr{Q}$ семейством $\mathscr{R}$ многозначного отображения $\mathscr{A}$, которое элементу $x \in \mathscr{Q}$ сопоставляет множество $\mathscr{A} x=\{y:(x, y) \in W\}$. В настояшее время имеется большое число работ, посвященных наилучшему приближению многозначных операторов (в частности, функционалов) однозначньми. Приведенные в этом параграфе результаты были даны в [5] именно для задачи (2.22).

\section{§ 3. Наилучшее приближение функционалов}

Приближение функционалов обладает существенными особенностями. В частности, для достаточно произвольных функционалов $A$, множеств $Q$ и классов приближающих функционалов $\mathscr{R}$ среди наилучших приближающих функционалов обязательно найдется линейный; в частности, в задаче (2.2) при $\delta>0$ имеет место равенство

$$
\mathscr{E}_{\delta}(\mathscr{O})=\mathscr{E}_{\delta}\left(X^{*}\right)=\omega(\delta)
$$


По-видимому, впервые подобное обстоятельство отметил С. А. Смоляк [40] (см. также работу Н. С. Бахвалова [41]) для задачи (2.20) при $\delta=0$. Более точно, результат состоит в том, что если $A$ - вещественный линейньй функционал, $Q$ - центрально симметричное выпуклое множество, $I x=\left\{f_{k}(x)\right\}_{k=1}^{m}$ - вектор значений конечного числа линейных функционалов $f_{k}$ на элементе $x \in Q, \mathscr{F}_{m}$ - множество всех вещественных функций, а $\mathscr{L}_{m}$ - множество линейных функций $m$ переменных на $\mathbb{R}^{m}$, то для задачи $(2.20)$ (при $\delta=0)$ справедливы равенства

$$
\mathscr{E}_{0}^{*}\left(\mathscr{F}_{m}\right)=\mathscr{E}_{0}^{*}\left(\mathscr{L}_{m}\right)=\sup \{|A x|: x \in Q, I x=0\}
$$

При $\delta>0$ подобный результат получили А. Г. Марчук и К. Ю. Осипенко [24]. Позднее А. Г. Марчук [25], а также Ш. Мичелли и Т. Ривлин [28] получили результат типа (3.2) в более общей ситуации произвольного линейного оператора $I$ при $\delta>0$, а также в некоторых случаях при $\delta=0$.

Равенство (3.1) обусловлено тем, что в задаче (0.2) для функционалов оценка Стечкина (1.6) точна. Последний факт составляет суть следуюшей теоремы В. Н. Габушина [10]; подобное утверждение (при некоторых ограничениях) можно извлечь из работы Петре [33].

TеОРема 3.1. Пусть A - линейный функиионал с областью определения $D(A) \subset X, Q$ есть центрально симметричное выпуклое множество из $D(A)$. Тогда при любом $N>0$

$$
E(N)=\sup \{\omega(\delta)-N \delta: \delta>0\},
$$

при этом в задаче (0.2) существует экстремальный функционал.

Следуюшая теорема показьвает, в частности, что если (для линейных функционалов) известно решение задачи Стечкина при любом $N>0$, то это позволяет вьписать решение соответствуюших некорректных задач $\mathscr{E}_{\delta}(\mathscr{O})$ и $\mathscr{E}_{\delta}\left(X^{*}\right)$ при всех $\delta>0$; здесь $X^{*}$ есть сопряженное пространство для $X$, т.е. пространство линейных ограниченных функционалов на $X$.

ТЕОРема 3.2. При условиях предъдущей теоремъ

$$
\mathscr{E}_{\delta}(\mathscr{O})=\mathscr{E}_{\delta}\left(X^{*}\right)=\ell(\delta)=\omega(\delta), \quad \delta>0,
$$

причем если $T_{0}$ - экстремальный функиионал в задаче (2.2), то он будет әкстремальныцм и в задаче (0.2), в которой $N$ есть субдифференциал (производная) функции $\omega(\delta)$ в соответствующей точке.

Это утверждение является следствием предыдущей теоремы и следующей леммы [4], справедливой не только для функционалов. 
Лемма 3.1. Если $\omega(\delta)$ - выпуклая на $[0, \infty)$ функиия, и в задаче $(0.2)$ оценка Стечкина (1.6) является точной, т.е. $E(N)=\sup \{\omega(\delta)-N \delta: \delta>0\} \forall N>0$, то для любого фиксированного $\delta>0$

$$
\mathscr{E}_{\delta}(\mathscr{O})=\mathscr{E}_{\delta}(\mathscr{L})=\omega(\delta)
$$

причем экстремальные операторы в задаче (2.2) и в задаче (0.2), в которой $N$ является субдифференциалом (производной) $\omega(\delta)$ при этом фиксированном $\delta$, совпадают.

В заключение параграфа сформулируем одно обшее утверждение из [5] для многозначных функционалов, из которого следуют многие известные результаты.

Теорема 3.3. Пусть $X$ - вещественное линейное пространство, $W$ - уравновешенное выпуклое множество в $X \times \mathbb{R}$. Тогда в задаче $(2.22)$

$$
\mathscr{E}(\mathscr{O}, W)=\mathscr{E}\left(X^{\#}, W\right)=\sup \{t:(0, t) \in W\}
$$

где $X^{\#}$ - множество всех (вещественных) линейных функиионалов на $X$.

Общим вопросам приближения функционалов посвящено много работ; кроме уже упомянутых отметим также работы [42]-[44].

\section{$\S$ 4. Наилучшее приближение операторов дифференцирования и неравенства между нормами производных для функций одного переменного на оси и полуоси}

1. $\mathrm{K}$ настоящему времени наиболее исследованной является задача Стечкина о наилучшем приближении оператора дифференцирования $D^{k}$ порядка $k$ на классе

$$
Q=Q_{r, p}^{n}=\left\{x \in L_{r}: x^{(n)} \in L_{p},\left\|x^{(n)}\right\|_{L_{p}} \leqslant 1\right\}
$$

$n$ раз дифференцируемых функций $(0 \leqslant k<n)$

$$
\begin{gathered}
E(N)=E_{n, k}(N)=\inf \left\{U(T):\|T\|_{L_{r} \rightarrow L_{q}} \leqslant N\right\}, \\
U(T)=\sup \left\{\left\|x^{(k)}-T x\right\|_{L_{q}}: x \in Q_{r, p}^{n}\right\},
\end{gathered}
$$

в пространствах $L_{\gamma}=L_{\gamma}(S), 1 \leqslant \gamma \leqslant \infty$, на числовой оси $S=\mathbb{R}$ и полуоси $S=\mathbb{R}_{+}$. Пространство $L_{\infty}=L_{\infty}(S)$ трактуется здесь следуюшим образом. Если $q=\infty$ или $r=\infty$, то $L_{\infty}(S)$ есть пространство $C=C(S)$ непрерьвных ограниченных функций на $S$ с равномерной нормой. Если же $p=\infty$, то $L_{\infty}(S)$ понимается как пространство измеримых, существенно ограниченных функций на $S$. Из соображений однородности легко получить [2] зависимость величины $E(N)$ от $N$; а именно, если выполнено условие

$$
\mu=k+1 / r-1 / q>0
$$




$$
E(N)=N^{-\gamma} E(1), \quad \gamma=(n-k+1 / q-1 / p) /(k+1 / r-1 / q) .
$$

В большинстве утверждений этого параграфа предполагается выполненным условие (4.3); оно исключает лишш некоторые вырожденные значения параметров.

Для задачи (4.2) соответствующая функция (1.2) имеет вид

$$
\omega(\delta)=\sup \left\{\left\|x^{(k)}\right\|_{L_{q}}:\|x\|_{L_{r}} \leqslant \delta,\left\|x^{(n)}\right\|_{L_{p}} \leqslant 1\right\}
$$

Если $\mu=n-1 / p+1 / r>0$, то, как нетрудно проверить,

$$
\omega(\delta)=K \delta^{\alpha}, \quad K=\omega(1), \quad \alpha=(n-k-1 / p+1 / q) /(n-1 / p+1 / r) .
$$

В силу этой формулы $K=\omega(1)$ есть наименьшая (наилучшая) константа в неравенстве между нормами производных функций (неравенстве Колмогорова)

$$
\begin{gathered}
\left\|x^{(k)}\right\|_{L_{q}} \leqslant K\|x\|_{L_{r}}^{\alpha}\left\|x^{(n)}\right\|_{L_{p}}^{\beta}, \\
\alpha=(n-k-1 / p+1 / q) /(n-1 / p+1 / r), \quad \beta=1-\alpha .
\end{gathered}
$$

Следующее утверждение принадлежит С. Б. Стечкину; оно является конкретизацией теоремы 1.1 для задачи (4.2).

Теорема 4.1. Если $k+1 / r-1 / q>0$, то величина $E(N)$ и наилучшая константа $K$ в (4.7)+(4.8) связань неравенством

$$
E(N) \geqslant \beta \alpha^{\alpha / \beta} K^{1 / \beta} N^{-\alpha / \beta}, \quad N>0 .
$$

2. Неравенства (4.7) впервые появились в работе Харди и Литтлвуда 1912 года [45]; они показали, что в равномерной норме на оси (т.е. в $C(-\infty, \infty))$ при всех $k, n(1 \leqslant k<n)$ имеет место неравенство (4.7) с некоторой конечной константой. Первые точные неравенства были получены Ландау [46] и Адамаром [47] при $n=2$. А.Н. Колмогоров [48] в 1939 году нашел точную константу в неравенстве (4.7) при $p=q=r=\infty$ (в равномерной норме) на оси $S=(-\infty, \infty)$ для всех $k, n(1 \leqslant k<n)$. С.-Надь [49] получил неравенство (4.7) с наилучшей константой в случае $n=1, p \geqslant 1$, $q>r>0$; это единственный случай, когда неравенство (4.7) исследовано для всех возможных значений параметров $p, q, r$.

В следуюшей теореме В. Н. Габушина даны необходимые и достаточные условия на параметры, при которых неравенство (4.7) имеет место с конечной константой, причем сделано это при естественных для данной задачи ограничениях $0<p, q, r \leqslant \infty$. Доказательство теоремы в полном объеме дано в [50] (при $p, q, r \geqslant 1-$ в [51]), другое доказательство - в [52]. 
ТеОрема 4.2. Пусть выполнены условия $0<p, q, r \leqslant \infty, 0 \leqslant k<n$, причем $q \neq p$, если $k=0$. Тогда для $S=\mathbb{R}$ или $S=\mathbb{R}_{+}$неравенство (4.7) с конечной константой имеет место в том и только том случае, когда параметры $\alpha, \beta$ заданы соотношениями (4.8) и выполнены оба условия

$$
\text { a) } \frac{n-k}{r}+\frac{k}{p} \geqslant \frac{n}{q}, \quad \text { b) } p \geqslant 1 \text {. }
$$

В приводимой таблице 1 сведены те случаи, когда в (4.7) выгислена наилучшая константа.

Отметим еще некоторые результаты, относящиеся к неравенствам (4.7). Если $x-$ экстремальная функция в (4.7), то экстремальной является также каждая из функций $a x\left(b t+t_{0}\right)$, здесь $a \neq 0, b \neq 0, t_{0} \in S$ для $S=(-\infty, \infty)$ и $a \neq 0, b>0, t_{0}=0$ для $S=[0, \infty)$. В [72] доказано, что если в (4.10а) строгое неравенство, то в неравенстве (4.7) существует экстремальная функция (более ранняя на эту тему - работа [73]). Во всех случаях, когда на оси $S=(-\infty, \infty)$ была найдена наилучшая константа в $(4.7)$ для значений параметров, обращающих условие (4.10а) в равенство, экстремальной функции нет, однако, можно построить экстремальную последовательность, исходя из некоторой периодической функции; эту периодическую функцию назьвают “идеальной” экстремальной функцией (см. работы [48], [54], [55], [63], [58], [59]). На полупрямой в равномерной норме неравенство (4.7) мало изучено. Шенберг и Каваретта [74] привели некоторые свойства экстремальной функции для этого случая; ранее А. Горны, А. Картан, А.П. Моторин, Ю.И. Любич, С.Б. Стечкин дали оценки сверху и снизу наилучшей константы (см. работу [75] и приведенную там библиографию). Результат Харди, Литтльвуда, Полиа $[54, \S 7.8]$ о точной константе в неравенстве (4.7) при $p=q=r=2, n=2, k=1$ на полуоси $S=[0, \infty)$ явился основой большого числа исследований; кроме уже процитированных [70], [71] отметим здесь еше работы Като [76], А.П.Буслаева [77], Эверитта с коллегами [78]-[83], Квонга, Зеттла [84] и др. Обзоры результатов, относяшихся к неравенству (4.7) и близким проблемам, имеются в [84], [85].

3. Первый точный результат в задаче (4.2) получил С. Б. Стечкин. Он дал [2], [1] решение задачи (4.2) в пространстве $C$ (т.е. при $p=q=r=\infty$ ) на оси и полуоси для значений $n=2$ и $n=3(1 \leqslant k<n)$; в частности, вьписал для этих случаев экстремальные операторы $T^{n, k}$. А именно, он показал, что на оси $S=(-\infty, \infty)$ экстремальными являются операторы

$$
\begin{aligned}
& \left(T_{h}^{2,1} x\right)(t)=\left(T_{h}^{3,1} x\right)(t)=\frac{x(t+h)-x(t-h)}{2 h}, \quad N=h^{-1}, \\
& \left(T_{h}^{3,2} x\right)(t)=\frac{x(t+h)-2 x(t)+x(t-h)}{h^{2}}, \quad N=4 h^{-2},
\end{aligned}
$$

а на полуоси $S=[0, \infty)$ таковьми являются операторы

$$
\begin{aligned}
& \left(T_{h}^{2,1} x\right)(t)=\frac{x(t+h)-x(t)}{h}, \quad N=2 h^{-1}, \\
& \left(T_{h}^{3,1} x\right)(t)=\frac{-8 x(t)+9 x(t+h)-x(t+3 h)}{6 h}, \quad N=3 h^{-1}, \\
& \left(T_{h}^{3,2} x\right)(t)=\frac{2 x(t)-3 x(t+h)+x(t+3 h)}{3 h^{2}}, \quad N=2 h^{-2} ;
\end{aligned}
$$


Таблица 1

\begin{tabular}{|c|c|c|c|c|c|}
\hline$n$ & $k$ & $q$ & $r$ & $p$ & Авторы \\
\hline \multicolumn{6}{|c|}{$S=(-\infty, \infty)$} \\
\hline 2 & 1 & $\infty$ & $\infty$ & $\infty$ & Адамар [47] \\
\hline $2<n \leqslant 5$ & & $\infty$ & $\infty$ & $\infty$ & Ю. Г. Боссе (Г.Е. Шилов) [53] \\
\hline произвольное & $0<k<n$ & $\infty$ & $\infty$ & $\infty$ & А. Н. Колмогоров [48] \\
\hline 1 & 0 & $\geqslant r$ & $>0$ & $\geqslant 1$ & С.-Надь [49] \\
\hline произвольное & $0<k<n$ & 2 & 2 & 2 & Харди, Литтльвуд, Полиа [54] \\
\hline произвольное & $0<k<n$ & 1 & 1 & 1 & Стейн [55] \\
\hline произвольное & $0 \leqslant k<n$ & $\infty$ & 2 & 2 & Л.В. Тайков [56] \\
\hline 2 & 1 & 2 & $\geqslant 1$ & $\frac{r}{r-1}$ & Харди, Литтлвуд, Полиа [54] \\
\hline 2 & 0,1 & $\infty$ & $>0$ & $\infty$ & В.Н. Габушин [57], \\
\hline 2 & 1 & $2 r$ & $\geqslant \frac{1}{2}$ & $\infty$ & В.Н. Габушин [58], А.П.Буслаев [59] \\
\hline 3 & 1,2 & $\frac{3 r}{3-k}$ & $\geqslant \frac{2}{3}$ & $\infty$ & В.Н. Габушин [58], А.П.Буслаев [59] \\
\hline 2 & 1 & $\geqslant 2 p$ & $\infty$ & $\geqslant 1$ & В.В. Арестов [60] \\
\hline 3 & 1,2 & $\infty$ & $\infty$ & $\geqslant 1$ & В.В. Арестов [60] \\
\hline 2 & 0 & $\infty$ & $>1$ & 1 & Г. Г. Магарил-Ильяев [61], [62] \\
\hline 3 & 1 & $\infty$ & $\geqslant 1$ & 1 & Г. Г. Магарил-Ильяев [61], [62] \\
\hline $2 k$ & $\geqslant 1$ & 2 & $\geqslant 1$ & $\frac{r}{r-1}$ & В.Г. Соляр [63] \\
\hline 2 & 1 & $\frac{2 r}{1+r}$ & $\geqslant 1$ & 1 & В.В.Арестов, В.И. Бердышев [64] \\
\hline \multicolumn{6}{|c|}{$S=[0, \infty)$} \\
\hline 2 & 1 & $\infty$ & $\infty$ & $\infty$ & Ландау [46] \\
\hline 3 & 1,2 & $\infty$ & $\infty$ & $\infty$ & А. П. Маторин [65] \\
\hline 2 & 1 & 2 & 2 & 2 & Харди, Литтльвуд, Полиа [54] \\
\hline 2 & 1 & $\geqslant 2 p$ & $\infty$ & $\geqslant 1$ & В.В. Арестов [60] \\
\hline произвольное & $0 \leqslant k<n$ & $\infty$ & 2 & 2 & В. Н. Габушин [66], Н. П. Купцов [67] \\
\hline 2 & 1 & 1 & 1 & 1 & В.И. Бердышев [68] \\
\hline 2 & 0,1 & $\infty$ & $>0$ & $\infty$ & Г.Г. Магарил-Ильяев [69], \\
\hline 2 & 0,1 & $\infty$ & $\geqslant 1$ & $\infty$ & Г.Г. Магарил-Ильяев [61], [62] \\
\hline 2 & 0 & $\infty$ & $\geqslant 1$ & 1 & Г. Г. Магарил-Ильяев [61], [62] \\
\hline 3 & 1 & $\infty$ & $\geqslant 1$ & 1 & Г. Г. Магарил-Ильяев [61], [62] \\
\hline произвольное & $0<k<n$ & 2 & 2 & 2 & Ю. И. Любич [70], Н. П. Купцов [71] \\
\hline 2 & 1 & $\frac{2 r}{1+r}$ & $\geqslant 1$ & 1 & В.В. Арестов, В. И. Бердышев [64] \\
\hline
\end{tabular}


здесь $h-$ положительный параметр.

Для оси $S=(-\infty, \infty)$ в пространстве $C(S)$ при $n=4,5$ решение задачи (4.2) дано в [86], [87]. Оно качественно отличается от предыдущих: здесь экстремальный оператор бесконечноразностный. Из результатов работы Домара [88] следует, что неравенство (4.9) в данном случае при всех $n$ обращается в равенство; из этой работы можно извлечь также определенную информацию об экстремальном операторе, в частности, что он является разностным. Экстремальный оператор в общем случае $(n>5)$ выписан в [89]. На полупрямой в равномерной норме задача Стечкина до сих пор мало изучена.

Случаи точного решения задачи (4.2) сведены в таблищу 2.

Таблица 2

\begin{tabular}{|c|c|c|c|c|c|}
\hline$n$ & $k$ & $q$ & $r$ & $p$ & Авторы \\
\hline \multicolumn{6}{|c|}{$S=(-\infty, \infty)$} \\
\hline 2,3 & $1 \leqslant k<n$ & $\infty$ & $\infty$ & $\infty$ & С.Б. Стечкин [2], [1] \\
\hline 4,5 & $1 \leqslant k<n$ & $\infty$ & $\infty$ & $\infty$ & В.В.Арестов [87] \\
\hline произволшное & $1 \leqslant k<n$ & $\infty$ & $\infty$ & $\infty$ & А.П. Буслаев [89] \\
\hline произволшное & $1 \leqslant k<n$ & 2 & 2 & 2 & Ю.Н. Субботин, Л.В. Тайков, [90] \\
\hline произвольное & $0 \leqslant k<n$ & $\infty$ & 2 & 2 & Л.В. Тайков [56] \\
\hline 2,3 & $1 \leqslant k<n$ & $\infty$ & $\infty$ & $\geqslant 1$ & В.В. Арестов [60] \\
\hline произволњное & $1 \leqslant k<n$ & 2 & 2 & 1 & В.В.Арестов [91] \\
\hline произвольное & $1 \leqslant k<n$ & 1 & 1 & 1 & В.В.Арестов [92] \\
\hline \multicolumn{6}{|c|}{$S=[0, \infty)$} \\
\hline 2,3 & $1 \leqslant k<n$ & $\infty$ & $\infty$ & $\infty$ & С.Б. Стечкин [2], [1] \\
\hline 2,3 & $1 \leqslant k<n$ & $\infty$ & $\infty$ & $\geqslant 1$ & В.В. Арестов [60] \\
\hline 2 & 1 & 1 & 1 & 1 & В. И. Бердышев [68] \\
\hline произвольное & $0 \leqslant k<n$ & $\infty$ & 2 & 2 & В. Н. Габушин [66] \\
\hline
\end{tabular}

Практически во всех перечисленных работах (за исключением [91]) использовалось неравенство С.Б. Стечкина (4.9). В некоторых случаях (см. [1], [2], [87], [93], [89], [90]) соответствуюшее точное неравенство (4.7) было известно ранее, в других же (см. [56], [66], [68], [60]) одновременно была найдена наименьшая константа $K$ в (4.7). В работе [91] для решения задачи использовалась специфика инвариантности относительно сдвига задачи (4.2); подробнее об этом см. параграф 6 данной работы. В дополнение к приведенной таблице отметим еще, что если известна точная константа и экстремальная функция в неравенстве Колмогорова при $q=\infty$, то, используя теоремы 3.1 и 3.2 В. Н. Габушина, можно сразу выписать точное решение задачи Стечкина.

Величина $E(N)$ не всегда конечна. В следующей теореме даны необходимые и достаточные условия ее конечности, причем сделано это для параметров $p, q, r$, 
удовлетворяющих более обшим, чем это до сих пор предполагалось, ограничениям $0<p, q, r \leqslant \infty$. Достаточность выписанных условий доказывается довольно просто [2], [94], [95]. Содержательньм в этой теореме является доказательство необходимости. Оно было дано в работах В. Н. Габушина [96], [97]; другое доказательство при $p, q, r \geqslant 1$ см. в [91]. Сушествование экстремального оператора доказано в [98], [4], сушествование инвариантного относительно сдвига (при $S=\mathbb{R}$ ) - в [4], [99], [6].

ТЕОРема 4.3. Если выполнены условия $0<p, q, r \leqslant \infty, 0 \leqslant k<n, u q \neq p$ при $k=0$, то (для $\left.S=\mathbb{R} u S=\mathbb{R}_{+}\right)$величина $E_{n, k}(N), N>0$, конечна тогда и только тогда, когда выполняется одно из условий

a) $q \geqslant r \geqslant 1, q \geqslant p \geqslant 1$,

b) $q=\infty, k=n-1, p=1$, причем $r<\infty$ для $k=0$.

Во всех случаях в задаче $E(N), N>0$, существует әкстремальный оператор, а для $S=\mathbb{R}$ среди әкстремальных операторов существует инвариантный относительно сдвига.

Как видно из теорем 4.2, 4.3, условия конечности величины $E_{n, k}(N), N>0$, и константы $K$ в неравенстве Колмогорова (4.7) различные. Важным и до сих пор нерешенным является вопрос о том, когда (при вьполнении условия (4.3), обеспечивающего конечность обеих величин $E(N)$ и $K)$ неравенство (4.9) обрашается в равенство; в этом случае будем говорить, что задачи Стечкина и Колмогорова согласованы. Для конкретных значений параметров, при которых была исследована задача (4.2) в работах [1], [2], [87], [90], [56], [66], [68], [60], [93], [89] задачи Стечкина и Колмогорова были согласованы. В следуюшей теореме, взятой из работы [6], приведены значения параметров, при которых задачи Стечкина и Колмогорова заведомо не согласованы.

Теорема 4.4. В следующих трех случаях

1) $q=r=2,1 \leqslant p<2,0<k<n$,

2) $q=p=2,1 \leqslant r<2,0 \leqslant k<n$,

3) $r=1,1 \leqslant q<\infty, 1<p \leqslant \infty, 0 \leqslant k<n$,

неравенство (4.9) является строгим.

Из сравнения приведенных вьше таблищ видно, что задача (4.2) решена в гораздо меншшем числе случаев в сравнении с тем, когда вычислена точная константа в неравенстве (4.7). Так, например, до сих пор неизвестно решение задачи Стечкина (4.2) в $L_{2}(0, \infty)$; здесь имеются лишш близкие между собой оценки сверху и снизу величины $E(N)$ при $n=2, k=1[100]$.

В работах [2], [101] решена задача о наилучшем приближении в пространстве $C$ на оси и полуоси оператора дифференцирования первого и второго порядка на классах функций с заданной мажорантой модуля непрерьвности первой и второй производной. В [102] найдена наилучшая константа в неравенстве Колмогорова (4.7) и решена задача Стечкина (4.2) в пространстве $C[0, \infty)$ для дробных производных малых порядков $k, n$.

4. Рассмотрим соответствуюшую некорректную задачу об оптимальном восстановлении значений оператора дифференцирования $D^{k}$ порядка $k$ на классе $Q=Q_{r, p}^{n}$ 
$n$ раз дифференцируемых функций:

$$
\mathscr{E}_{\delta}(\mathscr{R})=\mathscr{E}_{\delta}\left(\mathscr{R} ; D^{k}, Q_{r, p}^{n}\right)=\inf \left\{U_{\delta}\left(T ; D^{k}, Q_{r, p}^{n}\right): T \in \mathscr{R}\right\},
$$

$$
U_{\delta}(T)=U_{\delta}\left(T ; D^{k}, Q_{r, p}^{n}\right)=\sup \left\{\left\|f^{(k)}-T f_{\delta}\right\|_{L_{q}}: f \in Q_{r, p}^{n}, f_{\delta} \in L_{p},\left\|f-f_{\delta}\right\|_{L_{p}} \leqslant \delta\right\} ;
$$

это есть задача о наилучшем численном дифференцировании функций, заданных с ошибкой. Здесь мы предполагаем вновь, что $p, q, r \geqslant 1$.

Один из первых точных результатов в задаче (4.17) также принадлежит С. Б. Стечкину. Так, в его лекциях по наилучшему приближению неограниченных операторов ограниченными на Всесоюзной конференции по теории приближения в г. Махачкала в 1969 году содержалось, в частности, утверждение о том, что операторы (4.11)-(4.15) дают решение задачи (4.17) в пространстве $C$ на оси и полуоси для $n=2,3$. Например, для $n=2, k=1$ на оси $S=(-\infty,+\infty)$ имеют место равенства

$$
\mathscr{E}_{\delta}(\mathscr{O})=\mathscr{E}_{\delta}(\mathscr{L})=\sqrt{2 \delta},
$$

и оператор (4.11) при $h=\sqrt{2 \delta}$ является здесь экстремальным.

Нетрудно проверить, что при выполнении условия $(4.3)$ для величин $\mathscr{E} \delta(\mathscr{O})$ и $\mathscr{E} \delta(\mathscr{L})$ имеют место формулы, аналогичные (4.6):

$$
\mathscr{E}_{\delta}(\mathscr{O})=\mathscr{E}_{1}(\mathscr{O}) \delta^{\alpha}, \quad \mathscr{E}_{\delta}(\mathscr{L})=\mathscr{E}_{1}(\mathscr{L}) \delta^{\alpha}, \quad \delta>0
$$

Неравенство (2.11) в данном случае в силу результата С. И. Пичугова (2.8) и формулы (4.6) принимает вид

$$
K \delta^{\alpha} \leqslant \mathscr{E}_{\delta}(\mathscr{O}) \leqslant 2^{\frac{\theta-1}{\theta}} K \delta^{\alpha}, \quad \theta=\max \left(q, q^{\prime}\right), \quad 1 / q+1 / q^{\prime}=1
$$

В силу (4.9) для функций $\ell(\delta)$ и $\ell^{*}(\delta)$, определенных соотношениями $(1.5)$ и $(2.15)$, в рассматриваемом случае имеют место формулы

$$
\ell(\delta)=\ell(1) \delta^{\alpha}, \quad \ell^{*}(\delta)=\ell^{*}(1) \delta^{\alpha}
$$

При этом

$$
\ell(1)=\left(\frac{N}{\alpha}\right)^{\alpha}\left(\frac{E(N)}{1-\alpha}\right)^{1-\alpha}=\left(\frac{1}{\alpha}\right)^{\alpha}\left(\frac{E(1)}{1-\alpha}\right)^{1-\alpha} .
$$

С помощью формул $(2.13),(2.14)$ можно выписать явное выражение и для $\ell^{*}(1)$; так, при $2 \leqslant q \leqslant \infty$ имеем

$$
\ell^{*}(1)=E(1)^{1-\alpha}\left(\alpha^{-\alpha}(1-\alpha)^{\alpha-1}\right)^{1 / q}
$$

Таким образом, оценки $(2.17)$ величины $\mathscr{E}_{\delta}(\mathscr{L})$ в данной ситуации имеют вид

$$
\ell^{*}(1) \delta^{\alpha} \leqslant \mathscr{E}_{\delta}(\mathscr{L}) \leqslant \ell(1) \delta^{\alpha}
$$


В силу неравенств (4.19) и (4.21) теоремы 4.2 и 4.3 дают также необходимые и достаточные условия конечности величин $\mathscr{E}_{\delta}(\mathscr{O}), \mathscr{E}_{\delta}(\mathscr{L})$ наилучшего численного дифференцирования функций, заданных с погрешностью; эти условия различные и даны, соответственно, в (4.10) и (4.16). Важным является вопрос о том, когда (при выполнении условий (4.3)) имеет место равенство

$$
\mathscr{E}_{\delta}(\mathscr{O})=\mathscr{E}_{\delta}(\mathscr{L})
$$

Нетрудно убедиться, что если неравенство (4.9) обращается в равенство, то имеет место также равенство $\omega(\delta)=\ell(\delta)$, а это в силу (2.4) влечет за собой (4.22). В работах [1], [2], [87], [90], [56], [66], [68], [60], [93], [89] имела место именно эта ситуация. Поэтому в перечисленных работах наряду с решением задачи (4.2) и задачи о точном неравенстве Колмогорова (4.7) содержится также решение соответствуюших задач об оптимальном дифференцировании функций, заданных с погрешностью, а точнее, результаты этих работ позволяют выписать (даже если это и не было сделано) значение величин $\mathscr{E}_{\delta}(\mathscr{O})=\mathscr{E}_{\delta}(\mathscr{L})$ и наилучший (линейньй) метод, на котором в этих задачах достигается нижняя грань. Отметим еше, что из теорем 3.1 и 3.2 В. Н. Габушина можно сделать вывод, что равенство (4.22) имеет место при $q=\infty$ для произвольных значений остальных параметров. Поскольку неравенство (4.9) может быть и строгим, то, вероятно, и (4.22) будет иметь место не для всех значений параметров.

\section{$\S$ 5. Приближение операторов дифференцирования на классах функций многих переменных}

Задача Стечкина о наилучшем приближении операторов дифференцирования ограниченными операторами на классах функций многих переменных решена лишш в некоторых случаях. Здесь будут приведены наиболее интересные из них.

1. Пусть $W=W_{\infty}^{\left(n_{1}, n_{2}\right)}=W_{\infty}^{\left(n_{1}, n_{2}\right)}\left(\mathbb{R}^{2}\right)$ для натуральных $n_{1}, n_{2}$ есть множество вешественных функций $f(t)=f\left(t_{1}, t_{2}\right)$ двух переменных $t=\left(t_{1}, t_{2}\right)$, непрерывных и ограниченных на $\mathbb{R}^{2}$, т.е. принадлежаших пространству $C=C\left(\mathbb{R}^{2}\right)$, со следуюшими свойствами. Частная производная $f^{\left(n_{1}-1,0\right)}\left(t_{1}, t_{2}\right)$ функции $f \in W$ сушествует, локально абсолютно непрерывна по переменному $t_{1}$ на $\mathbb{R}$ почти для всех $t_{2} \in \mathbb{R}$, и производная $f^{\left(n_{1}, 0\right)}\left(t_{1}, t_{2}\right)$ существенно ограничена на $\mathbb{R}^{2}$, т.е. принадлежит пространству $L_{\infty}=L_{\infty}\left(\mathbb{R}^{2}\right)$. Аналогично, частная производная $f^{\left(0, n_{2}-1\right)}\left(t_{1}, t_{2}\right)$ функции $f \in W$ локально абсолютно непрерьвна по переменному $t_{2}$ на $\mathbb{R}$ для почти всех $t_{1} \in \mathbb{R}$ и производная $f^{\left(0, n_{2}\right)}$ принадлежит пространству $L_{\infty}\left(\mathbb{R}^{2}\right)$. Для функции $f \in W_{\infty}^{\left(n_{1}, n_{2}\right)}$ полагаем

$$
\|f\|=\|f\|_{C\left(\mathbb{R}^{2}\right)}, \quad\left\|f^{\left(n_{1}, 0\right)}\right\|=\left\|f^{\left(n_{1}, 0\right)}\right\|_{L_{\infty}\left(\mathbb{R}^{2}\right)}, \quad\left\|f^{\left(0, n_{2}\right)}\right\|=\left\|f^{\left(0, n_{2}\right)}\right\|_{L_{\infty}\left(\mathbb{R}^{2}\right)} .
$$

Здесь и ниже для пары $k=\left(k_{1}, k_{2}\right)$ неотрицательных целых чисел $k_{1}, k_{2}$ и функции $f$ двух переменных символом $f^{\left(k_{1}, k_{2}\right)}$ обозначается частная производная

$$
f^{\left(k_{1}, k_{2}\right)}\left(t_{1}, t_{2}\right)=\frac{\partial^{|k|} f\left(t_{1}, t_{2}\right)}{\partial t_{2}^{k_{2}} \partial t_{1}^{k_{1}}}, \quad|k|=k_{1}+k_{2} .
$$


В. Н. Коновалов [103] доказал, что на множестве функций $f \in W_{\infty}^{(3,3)}$ имеет место точное неравенство

$$
\left\|f^{(1,1)}\right\| \leqslant\left(3\|f\|\left\|f^{(3,0)}\right\|\left\|f^{(0,3)}\right\|\right)^{1 / 3}
$$

это неравенство обрашается в равенство на функциях $a f_{3}\left(b_{1} t_{1}+b_{2} t_{2}\right)$, где $a, b_{1}, b_{2}-$ отличные от нуля константы, а $f_{3}$ - сплайн Эйлера третьего порядка, являющийся, согласно результату Г.Е. Шилова [53], экстремальной функцией в неравенстве (4.7) в пространстве $C(-\infty, \infty)$ при $n=3$.

Неравенству (5.1) соответствует задача

$$
\begin{gathered}
E(N)=\inf \left\{U(T):\|T\|_{C \rightarrow C} \leqslant N\right\}, \\
U(T)=\sup \left\{\left\|f^{(1,1)}-T f\right\|_{C}: f \in Q_{\infty}^{(3,3)}\right\},
\end{gathered}
$$

о наилучшем приближении оператора дифференцирования

$$
D^{(1,1)}=\frac{\partial^{2}}{\partial t_{2} \partial t_{1}}
$$

ограниченными линейными операторами в пространстве $C=C\left(\mathbb{R}^{2}\right)$ на классе

$$
Q=Q_{\infty}^{(3,3)}=\left\{f \in W_{\infty}^{(3,3)}:\left\|f^{(3,0)}\right\| \leqslant 1,\left\|f^{(0,3)}\right\| \leqslant 1\right\}
$$

Эту задачу решил А.П. Буслаев [89]. Он доказал, что

$$
E(N)=\frac{2}{3} N^{-1 / 2}
$$

и оператор

$$
\begin{aligned}
\left(T_{h} f\right)\left(t_{1}, t_{2}\right)=\frac{1}{4 h^{2}}( & f\left(t_{1}+h, t_{2}+h\right)-f\left(t_{1}-h, t_{2}+h\right) \\
& \left.-f\left(t_{1}+h, t_{2}-h\right)+f\left(t_{1}-h, t_{2}-h\right)\right), \quad N=h^{-2}, h>0,
\end{aligned}
$$

является экстремальным в (5.2). Большой интерес представляет полученное О. А. Тимошиным [104] для функций $f \in W_{\infty}^{(3,3)}$ интегральное представление

$$
f^{(1,1)}-T_{h} f=f^{(3,0)} * G_{1}+f^{(0,3)} * G_{2},
$$

правая часть которого является суммой сверток производных $f^{(3,0)}$ и $f^{(0,3)}$ с суммируемьми на $\mathbb{R}^{2}$ функциями $G_{1}, G_{2}$, обладающими экстремальным свойством

$$
U\left(T_{h}\right)=\left\|G_{1}\right\|_{L\left(\mathbb{R}^{2}\right)}+\left\|G_{2}\right\|_{L\left(\mathbb{R}^{2}\right)} .
$$

Недавно О. А. Тимошин [105] получил решение задач, аналогичных (5.1), (5.2), при $n_{1}=3, n_{2}=2$ (или, то же самое, $n_{1}=2, n_{2}=3$ ). Именно, он доказал, что на классе функций $W^{(3,2)}$ имеет место точное неравенство

$$
\left\|f^{(1,1)}\right\| \leqslant\left(9\left\|f^{(3,0)}\right\|\right)^{1 / 3}\left(2\left\|f^{(0,2)}\right\|\right)^{1 / 2}\|f\|^{1 / 6} .
$$


Одновременно О. А. Тимошин решил аналогичную (5.2) задачу о наилучшем приближении оператора (5.3) ограниченньми линейными операторами в пространстве $C\left(\mathbb{R}^{2}\right)$ на классе

$$
Q=Q_{\infty}^{(3,2)}=\left\{f \in W_{\infty}^{(3,2)}:\left\|f^{(3,0)}\right\| \leqslant 3,\left\|f^{(0,2)}\right\| \leqslant 2\right\} .
$$

В данном случае

$$
E(N)=5 N^{-1 / 5},
$$

и оператор (5.5) вновь является экстремальным.

О. А. Тимошин выписал [104], [105] также решение соответствуюших некорректных задач

$$
\begin{gathered}
(5.8) \quad \mathscr{E}_{\delta}(\mathscr{R})=\mathscr{E}_{\delta}\left(\mathscr{R} ; D^{(1,1)}, Q\right)=\inf \left\{U_{\delta}\left(T ; D^{(1,1)}, Q\right): T \in \mathscr{R}\right\}, \\
U_{\delta}(T)=U_{\delta}\left(T ; D^{(1,1)}, Q\right)=\sup \left\{\left\|f^{(1,1)}-T f_{\delta}\right\|_{C}: f \in Q, f_{\delta} \in C,\left\|f-f_{\delta}\right\|_{C} \leqslant \delta\right\},
\end{gathered}
$$

об оптимальном вычислении значений оператора (5.3)на функциях классов $Q=Q_{\infty}^{(3,3)}$ и $Q=Q_{\infty}^{(3,2)}$ в пространстве $C$. В случае класса $Q=Q_{\infty}^{(3,3)}$ имеет место равенство

$$
\mathscr{E}_{\delta}(\mathscr{O})=\mathscr{E}_{\delta}(\mathscr{L})=(3 \delta)^{1 / 3},
$$

и (линейный ограниченный) оператор (5.5) со значением $h=(3 \delta)^{1 / 3}$ является оптимальным (экстремальным). Подобное утверждение получено им и для класса $Q=Q_{\infty}^{(3,2)}$.

В работах О. А. Тимошина [104], [105] доказано, что неравенства (5.1), (5.6) и формулы $(5.4),(5.7),(5.5),(5.9)$ для решений задач $(5.2),(5.8)$ о наилучшем приближении оператора (5.3) справедливы не только в пространствах $C, L_{\infty}$, но и в пространстве $L=L\left(\mathbb{R}^{2}\right)$.

2. В.Г. Тимофеев [106] получил для функций $f(t)=f\left(t_{1}, \ldots, t_{m}\right), m \geqslant 2$, переменных $t=\left(t_{1}, \ldots, t_{m}\right)$ точное неравенство

$$
\left\|\frac{\partial f}{\partial t_{i}}\right\|_{C\left(\mathbb{R}^{m}\right)} \leqslant \sqrt{2}\|f\|_{C\left(\mathbb{R}^{m}\right)}^{1 / 2}\|\Delta f\|_{L_{\infty}\left(\mathbb{R}^{m}\right)}^{1 / 2}, \quad i=\overline{1, m} ;
$$

здесь

$$
\Delta f=\frac{\partial^{2} f}{\partial t_{1}^{2}}+\cdots+\frac{\partial^{2} f}{\partial t_{m}^{2}}
$$

есть значение оператора Лапласа на функции $f$, определяемое по схеме Соболева. Неравенство (5.10) обрашается в равенство на одномерных функциях $a f_{2}\left(b t_{i}\right)$, где $a, b$ - отличные от нуля константы, а $f_{2}$ - экстремальная функция в неравенстве (4.7) в пространстве $C(-\infty, \infty)$ при $n=2$, доказанном Адамаром [47]. В [106] решена также соответствуюшая задача о наилучшем приближении в пространстве $C=C\left(\mathbb{R}^{m}\right)$ оператора дифференцирования $\partial / \partial t_{i}$ ограниченными линейными операторами на классе

$$
Q=\left\{f \in C\left(\mathbb{R}^{m}\right):\|\Delta f\|_{L_{\infty}\left(\mathbb{R}^{m}\right)} \leqslant 1\right\}
$$


и соответствующая некорректная задача оптимального восстановления значений оператора $\partial / \partial t_{i}$ на функциях класса $(5.11)$, заданных с погрешностью в пространстве $C$. В последних задачах экстремальный оператор является сверткой с некоторой мерой, сосредоточенной на гиперплоскостях $t_{i}= \pm h$. Как показано в [106], все эти результаты сохраняются также в пространстве $L\left(\mathbb{R}^{m}\right)$.

3. В настоящее время имеется ряд общих результатов, относящихся к задаче Стечкина о наилучшем приближении операторов дифференцирования на классах функций многих переменных из пространств Соболева и соответствующим родственным мультипликативным неравенствам между нормами производных в пространствах $L_{p}(S)$, $0<p \leqslant \infty$, для $S=\mathbb{R}^{m}$ и $S=\mathbb{R}_{+}^{m}$. Для оператора дифференцирования $D^{k}$, $k=\left(k_{1}, \ldots, k_{m}\right), k_{i} \in \mathbb{Z}_{+}$, определенного на функциях $f(t)=f\left(t_{1}, \ldots, t_{m}\right)$ формулой

$$
D^{k} f=\frac{\partial^{|k|} f}{\partial t_{1}^{k_{1}} \cdots \partial t_{m}^{k_{m}}}, \quad|k|=k_{1}+\cdots+k_{m},
$$

и класса функций

$$
\begin{aligned}
Q & =Q_{r, p}^{n}=Q_{r, p}^{n}(S) \\
& =\left\{f \in L_{p}(S):\left\|D^{i} f\right\|_{L_{r}(S)} \leqslant 1 \forall i=\left(i_{1}, \ldots, i_{m}\right),|i|=i_{1}+\cdots+i_{m}=n\right\}
\end{aligned}
$$

рассмотрим задачу Стечкина

$$
\begin{gathered}
E(N)=\inf \left\{U(T):\|T\|_{L_{r}(S) \rightarrow L_{q}(S)} \leqslant N\right\}, \\
U(T)=\sup \left\{\left\|D^{k} f-T f\right\|_{L_{q}(S)}: f \in Q_{r, p}^{n}(S)\right\},
\end{gathered}
$$

и мультипликативное неравенство между нормами производных

$$
\left\|D^{k} f\right\|_{L_{q}(S)} \leqslant K\|f\|_{L_{r}(S)}^{\alpha}\|f\|_{L_{p}^{n}(S)}^{\beta},
$$

где $\|f\|_{L_{p}^{n}(S)}=\max \left\{\left\|D^{i} f\right\|_{L_{p}(S)}:|i|=n\right\}$ - соболевская полунорма.

В предыдущем параграфе обсуждались все эти задачи при $m=1$. Многие общие результаты этого параграфа справедливы и при $m \geqslant 1$. В частности, соответствующая функция (1.2), имеющая в данной ситуации вид

$$
\omega(\delta)=\sup \left\{\left\|D^{k} f\right\|_{L_{q}(S)}:\|f\|_{L_{r}(S)} \leqslant \delta,\|f\|_{L_{p}^{n}(S)} \leqslant 1\right\}
$$

обладает такими свойствами. Если

$$
\mu=n-m / p+m / r \neq 0
$$

то, как нетрудно проверить, для $S=\mathbb{R}^{m}$ или $S=\mathbb{R}_{+}^{m}$ при $\delta>0$

$$
\omega(\delta)=K \delta^{\alpha}, \quad K=\omega(1), \quad \alpha=(n-|k|-m / p+m / q) /(n-m / p+m / r) .
$$


В силу этой формулы $K=\omega(1)$ есть наименьшая (наилучшая) константа в неравенстве (5.13) со значениями показателей

$$
\alpha=(n-|k|-m / p+m / q) /(n-m / p+m / r), \quad \beta=1-\alpha .
$$

Как и в одномерном случае, легко получить зависимость величины $E(N)$ от $N$; а именно, если выполнено условие $|k|+m / r-m / q \neq 0$, то

$$
E(N)=N^{-\gamma} E(1), \quad \gamma=\alpha / \beta=(n-|k|+m / q-m / p) /(|k|+m / r-m / q)
$$

Если $m \geqslant 1,|k|+m / r-m / q \neq 0$, то величина $E(N)$ и наилучшая константа $K$ в $(5.13)+(5.17)$ связаны неравенством

$$
E(N) \geqslant \beta \alpha^{\alpha / \beta} K^{1 / \beta} N^{-\alpha / \beta}, \quad N>0 .
$$

Это утверждение является конкретизацией теоремы 1.1 С.Б. Стечкина для задачи (5.12) и, очевидно, содержит теорему 4.1.

Неравенства между нормами производных (5.13) изучали Гальярдо, Ниренберг, К. К. Головкин, О. В. Бесов, В. П. Ильин, С. М. Никольский, Петре, В. Н. Габушин, В.М. Тихомиров, Г. Г. Магарил-Ильяев и многие другие (см. [107]-[110], [95], [50], [111]-[113], [7] и приведенную там библиографию). Окончательные (необходимые и достаточные) условия сушествования неравенства (5.13) с конечной константой (для $S=\mathbb{R}^{m}$ и $S=\mathbb{R}_{+}^{m}$ ) получил В.Н. Габушин; в большей части области изменения параметров они состоят в том, что

$$
\left(n-\frac{m}{p}+\frac{m}{r}\right)\left(\frac{n-|k|}{r}+\frac{|k|}{p}-\frac{n}{q}\right) \geqslant 0, \quad p \geqslant 1,
$$

причем $\alpha \geqslant 0$ при $\mu \neq 0$. Изложение этих результатов дано в [112] (случай $m=1-$ в [50]), другое доказательство дано в [113]; краткое изложение результатов см. в [114], [115], [7].

Величина $E(N)$, определенная в (5.12), конечна не для всех значений параметров. В настоящее время известны необходимые и достаточные условия ее конечности; в большей части области изменения параметров они состоят в том, что

$$
q \geqslant p \geqslant 1, \quad q \geqslant r \geqslant 1 \text {. }
$$

Достаточность выписанных условий доказывается довольно просто [2], [94], [95]. Содержательным является доказательство необходимости. Оно было дано в работах В.Н. Габушина [96], [97]; другое доказательство при $p, q, r \geqslant 1$ см. в [91].

Для соответствующей некорректной задачи $\mathscr{E}_{\delta}(\mathscr{R})=\mathscr{E}_{\delta}\left(\mathscr{R} ; D^{k}, Q_{r, p}^{n}\right)$ оптимального восстановления значений оператора $D^{k}$ на функциях класса $Q_{r, p}^{n}$, заданных с погрешностью $\delta$ в пространстве $L_{p}$, сохраняются соотношения (4.18)-(4.22).

4. Имеется еще ряд случаев, когда многомерные задачи, аналогичные обсуждаемым в этом параграфе, могут быть решены точно. Так, результаты работы [90] 
Ю.Н. Субботина, Л.В. Тайкова и работы [56] Л.В. Тайкова (полученные средствами гармонического анализа) для задачи (4.2) и неравенства (4.7) на оси, соответственно, при $p=q=r=2$ и $q=\infty, p=r=2$ без труда переносятся на многомерный случай. В работах [91], [116], [6] имеется ряд точных многомерных результатов, полученных с помошью соображений инвариантности; этому вопросу посвящен следующий параграф работы.

\section{§ 6. Приближение инвариантных операторов}

1. Если оператор $A$ и класс $Q$ инвариантны относительно некоторого семейства преобразований, то (при определенных, дополнительных условиях) в (0.2) и (2.2) можно ограничиться инвариантными операторами $T$, что в некоторых случаях существенно облегчает решение задач. Эта идея применялась и ранее, в частности, в задачах приближения классов функций, инвариантных относительно сдвига, с помошњю линейных методов (см. [117, гл. 7], [118, гл. 10]). Здесь используются следуюшие соображения. Пусть $T$ - произвольный линейный ограниченный оператор, к примеру, в пространстве $C_{2 \pi}$ непрерывных $2 \pi$-периодических функций, и $\tau_{h}$ - оператор сдвига. Тогда оператор $\widehat{T}$, определенный формулой

$$
\widehat{T} f=\frac{1}{2 \pi} \int_{0}^{2 \pi} \tau_{-h} T \tau_{h} f d h,
$$

линеен, инвариантен относительно сдвига, $\|\widehat{T}\| \leqslant\|T\|$ и $\widehat{T}$ аппроксимирует инвариантньй относительно сдвига класс функций не хуже, чем оператор $T$.

Операция усреднения оказалась полезной для исследования задачи Стечкина (4.2) о наилучшем приближении оператора дифференцирования. В работе [91] для приближения на оси и в работе [119] для периодического случая было доказано, что в (4.2) можно ограничиться операторами $T$, инвариантными относительно сдвига; для доказательства этого факта в [119] использовалась операция усреднения (6.1), а в [91] подобная операция для оси. Эти результаты позволили установить новые свойства $E(N)$, в частности, порядок $E(N)$ при $N \rightarrow \infty[119]$ и точное решение задачи (4.2) в некоторых случаях [91], [116], [6].

Более общая ситуация рассматривалась в работах [91], [116], [4], [99]. Допустим, что задачи (0.2), (2.2) обладают следующими свойствами инвариантности. Существуют полугруппа $S$ (операция в которой будет записываться мультипликативно) и семейства $\Theta=\{\theta(s), s \in S\}, \Upsilon=\{\tau(s), s \in S\}$ линейных ограниченных операторов в $X$ и $Y$, соответственно, являюшиеся полугруппами, т.е. обладаюшие свойствами

$$
\theta(s t)=\theta(s) \theta(t), \quad \tau(s t)=\tau(s) \tau(t), \quad t, s \in S
$$

такие, что для любых $s \in S$

$$
\begin{gathered}
\theta(s) Q \subset Q, \quad \tau(s) A \theta(s)=A, \\
\|\theta(s)\|_{X \rightarrow X} \leqslant 1, \quad\|\tau(s)\|_{Y \rightarrow Y} \leqslant 1 .
\end{gathered}
$$

Обозначим через $\mathscr{R}(S)$ множества инвариантных операторов из $\mathscr{R}$, т.е. операторов $T \in \mathscr{R}$ со свойством $\tau(s) T \theta(s)=T$ на $X$ для всех $s \in S$. Тогда при определенных 
дополнительных предположениях в задаче $(0.2)$ и в линейной задаче $(2.2)$ (т.е. в задаче $(2.2)$ для $\mathscr{R}=\mathscr{L})$ существует экстремальный оператор, обладающий свойством инвариантности, и, в частности, имеют место равенства

$$
E(N)=\inf \left\{U(T): T \in \mathscr{L}_{N}(S)\right\}, \quad \mathscr{E}_{\delta}(\mathscr{L})=\mathscr{E}_{\delta}(\mathscr{L}(S))
$$

К настоящему времени наиболее общие результаты такого типа содержатся в работе [99]; приводимая ниже теорема 6.1 взята именно из этой работы. Это утверждение будет относиться к задаче

$$
\begin{gathered}
\mathscr{E}(\mathscr{R}, W)=\inf \{U(T): T \in \mathscr{R}\}, \\
U(T)=U(T, W)=\sup \left\{\|y-T x\|_{Y}:(x, y) \in W\right\},
\end{gathered}
$$

(т.е. к задаче (2.22)) в предположении, что множество $W \subset X \times Y$ выпуклое и центрально симметричное, а следовательно, порожденное этой задачей многозначное отображение $x \in \mathscr{Q} \rightarrow \mathscr{A} x=\{y:(x, y) \in W\}$ также является выпуклым. Если множество $Q$ выпуклое и оператор $A$ линейный, то задачи $(0.2),(2.2)$ здесь, очевидно, содержатся.

Введем некоторые понятия и определения, которые позволят сформулировать необходимые ограничения на задачу.

Пусть $B=B(S)$ есть банахово пространство ограниченных вешественнозначных функций на полугруппе $S$ с нормой $\|f\|=\|f\|_{B(S)}=\sup \{|f(s)|: s \in S\}$. Функционал $m \in B^{*}$ называют средним на $B$, если значение $\langle m, f\rangle$ функционала $m$ на любой функции $f \in B$ удовлетворяет неравенствам

$$
\inf \{f(s): s \in S\} \leqslant\langle m, f\rangle \leqslant \sup \{f(s): s \in S\}
$$

Среднее $m$ называется левым инвариантным средним, если $\left\langle m,{ }_{t} f\right\rangle=\langle m, f\rangle$ для всех $t \in S, f \in B$; здесь ${ }_{t} f$ есть левая сдвижка функции $f$ на элемент $t$, определяемая формулой $\left({ }_{t} f\right)(s)=f(t s), s \in S$. Если на $B(S)$ сушествует левое инвариантное среднее, то полугруппа $S$ назьвается левой аменабельной. Свойства аменабельных полугрупп и инвариантных средних на них можно найти, например, в [120], [121]; в частности, известно, что коммутативная полугруппа является аменабельной.

Будем говорить, что задача (6.6) инвариантна, а точнее, $(\Theta, \Upsilon)$-инвариантна, если для любых $(x, y) \in W$ и $s \in S$ сушествует элемент $y^{\prime} \in Y$ со свойствами $y=\tau(s) y^{\prime}$, $\left(\theta(s) x, y^{\prime}\right) \in W$. Так, при выполнении условий (6.3) задачи (0.2) и (2.2) инвариантны.

ТЕОРема 6.1. Допустим, что выполнены следующие предположения.

1) Мнохество $W \subset X \times Y$ в задаче (6.6) выпуклое и иентрально симметричное.

2) Семейства $\Theta=\{\theta(s), s \in S\}, \Upsilon=\{\tau(s), s \in S\}$ являются полугруппами линейных ограниченных операторов в $X$ и $Y$, соответственно, со свойствами (6.4).

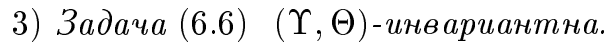

4) Полугруппа $S$ левая аменабельная. 
5) Существует проектор $\pi$ с единичной нормой из пространства $Y^{* *}$, второго сопряжснного для $Y$, в пространство $Y$, и при любом $s \in S$ второй сопряженный оператор $\tau^{* *}$ для оператора $\tau=\tau(s)$ коммутирует $c \pi$, m.e. $\tau^{* *} \pi=\pi \tau$.

6) $\mathscr{R}$ есть одно из множеств: множество $\mathscr{O}(\mathscr{Q}, Y)$ всех операторов из $\mathscr{Q}$ в $Y$, множество $\mathscr{L}(X, Y)$ линейных операторов, множество $\mathscr{B}(X, Y)$ линейных ограниченных операторов из $X$ в $Y$, множество $\mathscr{L}(N)=\mathscr{L}(N ; X, Y)=\{T \in \mathscr{B}$ : $\|T\| \leqslant N\}$ операторов из $\mathscr{B}$, нормы которых ограничены числом $N>0$.

Тогда имеет место равенство

$$
\mathscr{E}(\mathscr{R}, W)=\mathscr{E}(\mathscr{R}(S), W)
$$

и в задаче (6.6) существует әкстремальный оператор, являющийся $(\Upsilon, \Theta)$-инвариантным.

Отметим, что, если $Y$ - рефлексивное пространство, то предположение 5) вьполняется для любого семейства операторов $\Upsilon$.

2. Только что изложенные соображения оказались особенно полезными для изучения задачи наилучшего приближения в пространствах $L_{\gamma}\left(\mathbb{R}^{m}\right), 1 \leqslant \gamma \leqslant \infty$, неограниченных операторов, инвариантных относительно (любого) сдвига, каковыми являются, в частности, дифференциальные операторы с постоянными коэффициентами. Приводимые ниже результаты взяты из работ [91], [116], [6].

Пусть $\mathscr{S}=\mathscr{S}\left(\mathbb{R}^{m}\right)$ есть множество бесконечно дифференцируемых и быстро убываюших функций на $\mathbb{R}^{m}$, а $\mathscr{S}^{\prime}$ - соответствуюшее двойственное пространство обобщенных функций. Для элемента $a \in \mathscr{S}^{\prime}$ обозначим через $\bar{a}$ элемент из $\mathscr{S}^{\prime}$, действующий по правилу $\langle\bar{a}, x\rangle=\langle a, \bar{x}\rangle, \bar{x}(t)=x(-t), x \in \mathscr{S}$. Определим оператор $A$ с областью определения $D(A)$, лежащей в пространстве $\Sigma=\Sigma\left(\mathbb{R}^{m}\right)$ функций $x$, локально суммируемых и удовлетворяющих условию

$$
\int_{\mathbb{R}^{m}}(1+|t|)^{\mu}|x(t)| d t<\infty, \quad \mu=\mu(x) \leqslant 0
$$

следующим образом. На множестве $\mathscr{S}$ зададим $A$ в виде свертки $A x=\bar{a} * x$, а на $D(A)$ распространим оператор $A$ по схеме С. Л. Соболева, т.е. относительно пары функций $x, y \in \Sigma$ считаем, что $x \in D(A)$ и $y=A x$, если

$$
\int x A^{*} \phi d t=\int y \phi d t
$$

для любого $\phi \in \mathscr{S}$, где $A^{*}$ есть оператор, сопряженный для оператора $A$ и определяемьй на $\mathscr{S}$ формулой $A^{*} \phi=a * \phi$. При этом предполагается, что $а$ удовлетворяет условию $A^{*} \mathscr{S} \subset \mathscr{S}$, или, то же самое, $A \mathscr{S} \subset \mathscr{S}$ (для этого, например, достаточно, чтобы функционал $a$ имел компактньй носитель). С помощью еще одного элемента $b \in \mathscr{S}^{\prime}$ определим подобным же образом оператор $B$ с областью определения $D(B) \subset \Sigma$. Введем функциональньй класс

$$
Q=Q_{r, p}(B)=\left\{x \in L_{r} \cap D(B): B x \in L_{p},\|B x\|_{p} \leqslant 1\right\}
$$


Рассматривается величина

$$
E(N)=E(N ; r, s ; p, q)=\inf \left\{U(T): T \in \mathscr{L}\left(N ; L_{r}, L_{s}\right)\right\}
$$

где

$$
U(T)=U(T, Q)=\sup \left\{\|A x-T x\|_{L_{q}}: x \in Q\right\},
$$

а $\mathscr{L}\left(N ; L_{r}, L_{s}\right)=\mathscr{L}(N)$ - множество линейных ограниченных операторов $T$ из $L_{r}$ в $L_{s}$ с $\|T\| \leqslant N$. Если $r$ или $s$ есть $\infty$, то предполагается, что соответствующее пространство есть пространство $C_{0}$ функций, непрерывных и имеющих нулевой предел на бесконечности. Задача (6.8) инвариантна относительно группы сдвигов $\tau_{s}, s \in \mathbb{R}^{m}$, определенных формулой $\left(\tau_{s} x\right)(t)=x(t-s)$. Точнее, для оператора $A$, класса $Q$ и преобразований $\theta(s)=\tau_{s}, \tau(s)=\tau_{-s}, s \in \mathbb{R}^{m}$, вьполняются условия $(6.3),(6.4)$.

Обозначим через $\mathscr{T}_{r}^{s}(N)$ множество операторов $T \in \mathscr{L}\left(N ; L_{r}, L_{s}\right)$, инвариантных относительно всех сдвигов (на $L_{r}$ ). Следующее утверждение доказано в [116], [6]; оно содержится, вообще говоря, в предыдущей теореме 6.1 .

ТеОрема 6.2. При сделанных предположсениях относительно операторов $A$ и В, для любого $N>0$ имеет место равенство

$$
E(N)=\inf \left\{U(T): T \in \mathscr{T}_{r}^{s}(N)\right\}
$$

Множество $\mathscr{T}_{r}^{s}$ ограниченных операторов из $L_{r}$ в $L_{s}$, инвариантных относительно сдвига, достаточно хорошо изучено (см., например, монографии [122], [123]). Известно, что если $s<r$, то $\mathscr{T}_{r}^{s}$ состоит только из нулевого оператора, и потому в этом случае $E(N)=\sup \left\{\|A x\|_{L_{q}}: x \in Q\right\}$. При $q<p$ справедливо некоторое аналогичное утверждение. Эти два факта, как правило, влекут за собой (см. [91], [116], [6]), что если $s<r$ или $q<p$, то $E(N)=\infty$ при любом $N>0$. Таким образом, если $s<r$ или $q<p$, то задача (6.8) является вырожденной. В связи с этим будем считать, что $s \geqslant r$, $q \geqslant p$. Известно, что $\mathscr{T}_{r}^{s}=\mathscr{T}_{s^{\prime}}^{r^{\prime}}$ и $\|T\|_{L_{r} \rightarrow L_{s}}=\|T\|_{L_{s^{\prime}} \rightarrow L_{r^{\prime}}}, T \in \mathscr{T}_{r}^{s}=\mathscr{T}_{s^{\prime}}^{r^{\prime}}$, поэтому (при некоторых дополнительных предположениях) оказывается, что [91], [116], [6]

$$
E(N ; r, s ; p, q)=E\left(N ; s^{\prime}, r^{\prime} ; p, q\right)=E\left(N ; r, s ; q^{\prime}, p^{\prime}\right)=E\left(N ; s^{\prime}, r^{\prime} ; q^{\prime}, p^{\prime}\right)
$$

Оператор $T \in \mathscr{T}_{r}^{s}$ имеет вид свертки $T x=\bar{\theta} * x$, где $\theta=\theta_{T} \in \mathscr{S}^{\prime}$. Множество $M_{r, s}=M(r, s)$ таких обобщенных функций $\theta_{T}$ является банаховым пространством относительно нормы $\left\|\theta_{T}\right\|_{M_{r, s}}=\|T\|_{L_{r} \rightarrow L_{s}}$. Пространства $M(r, s)$, называемые пространствами мультипликаторов, довольно хорошо изучены (см. монографии [122]-[124]). Так, известно, что

$$
M(r, \infty)=L_{r^{\prime}}, \quad 1 \leqslant r<\infty, \quad M(\infty, \infty)=M(1,1)=\mathscr{V}
$$

где $\mathcal{V}$ - пространство ограниченных борелевских мер на $\mathbb{R}^{m}$.

Пространство $M_{r, s}$ является сопряженным для банахова пространства функций $L_{r, s}=L(r, s)$, построенного следуюшим образом (см. [123] и приведенную там 
библиографиюю). Если $1 \leqslant r \leqslant s<\infty$, то $L_{r, s}$ состоит из функций $h \in L_{\omega}$, $1 / \omega=1 / r-1 / s$, вида

$$
h=\sum x_{j} * y_{j}, \quad x_{j} \in L_{r}, y_{j} \in L_{s^{\prime}}, \quad \sum\left\|x_{j}\right\|_{r}\left\|y_{j}\right\|_{s^{\prime}}<\infty,
$$

и норма в $L_{r, s}$ определяется формулой

$$
\|h\|_{r, s}=\|h\|_{L_{r, s}}=\inf \sum\left\|x_{j}\right\|_{r}\left\|y_{j}\right\|_{s^{\prime}}
$$

где нижняя грань берется по всем представлениям (6.12). Если $s=\infty$, то, как нетрудно увидеть из (6.11), следует взять

$$
L_{\infty, \infty}=C_{0}, \quad L_{r, \infty}=L_{r}, \quad 1 \leqslant r<\infty .
$$

Во всех случаях $L_{r, s}$ является подпространством в $L_{\omega}, 1 / \omega=1 / r-1 / s$, содержит множество $L_{r} * L_{s^{\prime}}=\left\{x * y: x \in L_{r}, y \in L_{s^{\prime}}\right\}$, и при этом

$$
\begin{gathered}
\|x\|_{\omega} \leqslant\|x\|_{r, s}, \quad x \in L_{r, s}, \\
\|x * y\|_{r, s} \leqslant\|x\|_{r}\|y\|_{s^{\prime}}, \quad x \in L_{r}, y \in L_{s^{\prime}} .
\end{gathered}
$$

Наряду с операторной задачей (6.8) рассмотрим следуюшие функциональные задачи. Для функционала $\theta \in M_{r, s}$ положим

$$
u(\theta)=\sup \left\{\langle a, x\rangle-\langle\theta, x\rangle: x \in Q_{r, s ; p, q}(B)\right\},
$$

где

$$
Q_{r, s ; p, q}(B)=\left\{x \in L_{r, s}: B x \in L_{p, q},\|B x\|_{p, q} \leqslant 1\right\},
$$

и введем величину

$$
e(N)=\inf \left\{u(\theta):\|\theta\|_{M_{r, s}} \leqslant N\right\}
$$

наилучшего приближения функционала $а$ ограниченными функционалами. Далее, введем модуль непрерывности

$$
\omega_{0}(\delta)=\sup \left\{|\langle a, x\rangle|:\|x\|_{r, s} \leqslant \delta,\|B x\|_{p, q} \leqslant 1\right\}
$$

функционала $a$ на классе $Q_{r, s ; p, q}(B)$ и функцию

$$
\Delta_{0}(N)=\sup \left\{\omega_{0}(\delta)-N \delta: \delta>0\right\} .
$$

С помощью теоремы 6.2 можно обосновать следующие два утверж дения [6]. 
Теорема 6.3. Если выполнено одно из двух условий

1) оператор В обладает свойством

$$
\{g \in C, B g=0\} \Rightarrow g=0
$$

2) $p>1$ и оператор $B$ обладает свойством

$$
\left\{g \in C_{0}, B g=0\right\} \Longrightarrow g=0
$$

то при любом $N>0$

$$
E(N)=e(N)
$$

Поскольку предполагается, что $A S \subset S, B S \subset S$, можно говорить о свертке элементов $\zeta \in S^{\prime}$ с элементами $a$ и $b$. Допустим, что существует фундаментальная функция $\kappa$ оператора $B^{*}$, т.е. сушествует элемент $\kappa \in \mathscr{S}^{\prime}$ такой, что $\kappa * b$ есть $\delta$-функция. В этом случае

$$
a=\mu * b
$$

где $\mu=a * \kappa=A^{*} \kappa$. Обозначим через $M_{r, s}\left(B^{*}, N\right)$ множество элементов $\zeta \in \mathscr{S}^{\prime}$ таких, что $B^{*} \zeta=\zeta * b \in M_{r, s}$ и $\left\|B^{*} \zeta\right\|_{M_{r, s}} \leqslant N$. Введем величину

$$
\varepsilon(N)=\inf \left\{\|\mu-\zeta\|_{M_{p, q}}: \zeta \in M_{r, s}\left(B^{*}, N\right)\right\}
$$

наилучшего приближения элемента $\mu=A^{*} \kappa$ множеством элементов $M_{r, s}\left(B^{*}, N\right)$ в пространстве (мультипликаторов) $M_{p, q}$.

ТЕОРема 6.4. Если въполнено одно из двух условий предъдущей теоремьи и, кроме того, имеет место представление (6.22), то справедливы равенства

$$
E(N)=e(N)=\varepsilon(N) .
$$

Используя результат работы В. Н. Габушина [10], приведенньй вьше в теореме 3.1 , можно утверждать, что $e(N)=\Delta_{0}(N)$, и, значит, в условиях последней теоремы

$$
E(N)=e(N)=\varepsilon(N)=\Delta_{0}(N)
$$

Равенства (6.25) сводят решение операторной задачи (6.8) к более простым функциональньм задачам (6.16), (6.17), (6.23).

3. Рассмотрим более подробно наилучшее приближение оператора $A=D^{k}=$ $d^{k} / d t^{k}$ впространстве $L_{q}=L_{q}(-\infty, \infty)$ на числовойпрямоймножеством $\mathscr{L}\left(N ; L_{r}, L_{s}\right)$ линейных ограниченных операторов из $L_{r}$ в $L_{s}$ на классе

$$
Q_{r, p}^{n}=\left\{x \in L_{r}: x^{(n)} \in L_{p},\left\|x^{(n)}\right\|_{p} \leqslant 1\right\}
$$


Таким образом, здесь

$$
\begin{gathered}
E(N)=E_{n, k}(N)=E_{n, k}(N ; r, s ; p, q)=\inf \left\{U(T): T \in \mathscr{L}\left(N ; L_{r}, L_{s}\right)\right\}, \\
U(T)=\sup \left\{\left\|x^{(k)}-T x\right\|_{q}: x \in Q_{r, p}^{n}\right\} .
\end{gathered}
$$

Отметим, что в (6.26) в сравнении с (4.2) не предполагается, что $s=q$.

За исключением некоторых вырожденных значений параметров, в дополнение к теореме 4.3, можно утверждать [91], [6], что (при $p, q, r, s \geqslant 1$ ) величина $E_{n, k}(N)$, $N>0$, конечна тогда и только тогда, когда $s \geqslant r, q \geqslant p$, и, если $k+1 / r-1 / s>0$, то имеет место формула

$$
E_{n, k}(N)=N^{-\gamma} E_{n, k}(1), \quad \gamma=\frac{n-k+1 / q-1 / p}{k+1 / r-1 / s} .
$$

Операторы $A=D^{k}$ и $B=D^{n}$ являются операторами свертки, оператор $B$ имеет (классическую) характеристическую функцию

$$
\kappa_{n}(t)=\frac{t_{+}^{n-1}}{(n-1) !}, \quad t_{+}=\max (0, t)
$$

и $A \kappa_{n}=\kappa_{n}^{(k)}=\kappa_{n-k}$. Поэтому $(6.23)$ в данном случае является величиной

$$
\varepsilon_{n, k}(N)=\inf \left\{\left\|\kappa_{n-k}-\zeta\right\|_{M_{p, q}}: \zeta \in S^{\prime}, \zeta^{(n)} \in M_{r, s},\left\|\zeta^{(n)}\right\|_{M_{r, s}} \leqslant N\right\}
$$

наилучшего приближения в пространстве мультипликаторов $M_{p, q}$ классической функции $\kappa_{n-k}$ указанным классом обобшенных функций $\zeta$.

В данном случае (6.17) имеет вид

$$
\omega_{0}(\delta)=\sup \left\{\left|x^{(k)}(0)\right|:\|x\|_{r, s} \leqslant \delta,\left\|x^{(n)}\right\|_{p, q} \leqslant 1\right\} .
$$

Нетрудно проверить, что если $n+1 / q-1 / p+1 / r-1 / s>0$, то

$$
\begin{gathered}
\omega_{0}(\delta)=K \delta^{\alpha}, \quad K=\omega_{0}(1), \quad \alpha=\frac{n-k+1 / q-1 / p}{n+1 / q-1 / p+1 / r-1 / s} \\
\Delta_{0}(N)=\beta \alpha^{\alpha / \beta} K^{1 / \beta} N^{-\alpha / \beta}, \quad \beta=1-\alpha .
\end{gathered}
$$

Из (6.29) следует,что $K=\omega_{0}(1)$ является наименьшей константой в неравенстве

$$
\left\|x^{(k)}\right\|_{C} \leqslant K\|x\|_{r, s}^{\alpha}\left\|x^{(n)}\right\|_{p, q}^{1-\alpha}
$$

Оператор $B=D^{n}$ удовлетворяет условию (6.20) теоремы 4.3, поэтому эта теорема содержит следующее утверждение. 
ТЕОРемА 6.5. Если $s \geqslant r \geqslant 1, q \geqslant p>1$, причем $s>r$ nрu $k=0$, то для любого значения $N>0$

$$
E_{n, k}(N)=\varepsilon_{n, k}(N)=\beta \alpha^{\alpha / \beta} K^{1 / \beta} N^{-\alpha / \beta},
$$

где $K$ - наименьшая константа в (6.30).

Таким образом, величина $E_{n, k}(N)$ выражается через наименьшую константу в неравенстве (6.30), и, к тому же, при $q=s$ в силу (4.9) оценивает сверху наилучшую константу в неравенстве Колмогорова (4.7). Приведенная выше теорема 4.4 была доказана в [6] с помощью теоремы 6.5 и результата работы [72] о существовании экстремальной функции в неравенстве (4.7).

При определенных условиях на параметры удается (см. [91], [116], [6]) найти решение всех задач в (6.31). Так, в [116] это сделано при $p=q=2,1 \leqslant r=s \leqslant \infty$. Приведем соответствуюшее утверждение (6.30) для случая $r=s=\infty$. Можно утверждать, что если функция $x$ принадлежит пространству $C=C(-\infty, \infty)$ и преобразование $Ф$ рье $\widehat{x}^{(n)}$ производной $x^{(n)}$ имеет ограниченную вариацию $V \widehat{x}^{(n)}$, то при $1 \leqslant k<n$ справедливо точное неравенство

$$
\left\|x^{(k)}\right\|_{C} \leqslant K_{n, k}\|x\|_{C}^{\frac{n-k}{n}}\left(V \widehat{x}^{(n)}\right)^{\frac{k}{n}}
$$

с наименьшей константой $K_{n, k}=1$ при $n \geqslant 3$ и

$$
K_{2,1}=\left(\frac{32}{\pi^{3}} \sum_{\nu=0}^{\infty} \frac{1}{(2 \nu+1)^{3}}\right)^{1 / 2}>1
$$

В работе [125] была решена соответствуюшая неравенству (6.32) задача типа (4.2) о наилучшем приближении оператора дифференцирования порядка $k$ в пространстве $C(-\infty, \infty)$ на классе $Q=\left\{x \in C: V \widehat{x}^{(n)} \leqslant 1\right\}$.

\section{§ 7. Приближение одного класса дифференцируемых функций другим классом более гладких функций}

Задача (1.2) о вычислении модуля непрерывности линейного оператора и задача Стечкина (0.2) (двойственно) связаны с задачами наилучшего и наилучшего линейного приближения одного класса элементов другим в сопряженных пространствах [126]. В частности, имеет место двойственность задачи о наименьшей константе в неравенстве (4.7) с задачей о наилучшем приближении одного класса дифференцируемых функций другим подобным классом более гладких функций и двойственность задачи Стечкина (4.2) с задачей линейного приближения класса классом [127]; здесь они будут приведены для оси $S=\mathbb{R}=(-\infty, \infty)$.

Пусть $p^{\prime}, r^{\prime}, q^{\prime}$ - параметры, удовлетворяющие условию $1 \leqslant p^{\prime}, r^{\prime}, q^{\prime} \leqslant \infty$ и не связанные пока как-либо с параметрами $p, r, q ; l, n$ - целые числа, $0 \leqslant l \leqslant n ; B_{r^{\prime}}^{n}(N)$ множество функций $\varphi$, у которых $\left\|\varphi^{(n)}\right\|_{L_{r^{\prime}}} \leqslant N ; B_{q^{\prime}}^{l}=B_{q^{\prime}}^{l}(1)$. Для функции $\psi \in B_{q^{\prime}}^{l}$ положим

$$
F(\psi, N)=\inf \left\{\|\psi-\varphi\|_{L_{p^{\prime}}}: \varphi \in B_{r^{\prime}}^{n}(N)\right\}
$$


и введем величину

$$
F(N)=\sup \left\{F(\psi, N): \psi \in B_{q^{\prime}}^{l}\right\}
$$

наилучшего приближения в $L_{p^{\prime}}(-\infty, \infty)$ класса $B_{q^{\prime}}^{l}$ классом $B_{r^{\prime}}^{n}(N)$. Соответствующую величину линейного приближения класса классом будем обозначать через $G(N)$.

Задача приближения одного класса функций другим является классической для теории приближения (см., например, монографии В. М. Тихомирова [118], Н. П. Корнейчука [128]). Тот факт, что задача (7.1) и соответствующая линейная задача связаны с задачами (4.2) и (4.7), был замечен на Семинаре С. Б. Стечкина в Свердловском отделении Математического института им. В.А. Стеклова (ньне Институт математики и механики УрО РАН) в 1965-1967 годах. Понимание этой связи, впрочем, и развитие всей тематики, во многом было осуществлено участниками семинара Л. В. Тайковым, Ю. Н. Субботиным, Н. И. Черных, В. Н. Габушиным, В. И. Бердьшевым, автором и, конечно, руководителем семинара С. Б. Стечкиным.

Относительно величин $F(N)$ и $G(N)$ известно [129], что

$$
\begin{gathered}
F(N)=N^{-\gamma} F(1), \quad G(N)=N^{-\gamma} G(1), \\
\gamma=\left(l+1 / p^{\prime}-1 / q^{\prime}\right) /\left(n-l+1 / q^{\prime}-1 / r^{\prime}\right),
\end{gathered}
$$

и $F(N)$ конечная в том [130] и только в том (см. результат В. Н. Габушина в [129]) случае, если

$$
\frac{l}{r^{\prime}}+\frac{n-l}{p^{\prime}} \leqslant \frac{n}{q^{\prime}}
$$

В работах Ю.Н. Субботина [131], Ю.Н. Субботина и Л. В. Тайкова [90], автора и В. Н. Габушина [129] были найдены $F(N)$ и $G(N)$ для конкретных значений параметров. При этом для оценки сверху использовались следующие соображения, впервые приведенные в работе Л.В. Тайкова [132]. Допустим, что $l=n-k, p=r=q^{\prime}$, $p^{\prime}=r^{\prime}=q$ и задача (4.2) имеет экстремальньй оператор, которьй определен, линеен и перестановочен со сдвигом на множестве $\mathscr{W}_{p}^{n}$ функций $x$, у которых $x^{(n)} \in L_{p}$. Тогда

$$
F(N) \leqslant G(N) \leqslant E(N)
$$

В цитированных работах приводилась оценка $F(N)$ снизу, совпадающая с $E(N)=$ $E_{n, k}(N)$, что давало значение $F(N)=G(N)$. Этот подходпозволяет выгислить $F(N)$ и $G(N)$ лишш в некоторых, исключительных случаях. Приводимые ниже утверждения из [127] дают правильные, двойственные соотношения между рассматриваемыми задачами.

TEOpемa 7.1. Пусmь $1 \leqslant p, q, r \leqslant \infty$,

$$
l=n-k, \quad 1 / p+1 / p^{\prime}=1, \quad 1 / r+1 / r^{\prime}=1, \quad 1 / q+1 / q^{\prime}=1
$$

и выполнень условия

$$
\frac{n-k}{r}+\frac{k}{p} \geqslant \frac{n}{q}
$$


причем $q>r$, если $k=0$. Тогда при любом $N>0$ имеет место равенство

$$
F(N)=\beta \alpha^{\alpha / \beta} K^{1 / \beta} N^{-\alpha / \beta},
$$

где $K$ - начменьшая константа в (4.7), а параметры $\alpha$ и $\beta$ определены в (4.8).

Напомним, что условие (7.5) является условием конечности константы $K$ и величины $F(N)$.

Теорема 7.2. Допустим, что $1<q, p \leqslant \infty, 1 \leqslant r \leqslant \infty$ и выполнены условия (7.4). Тогда при любом $N>0$

$$
G(N)=E(N)
$$

В случае $q=\infty\left(q^{\prime}=1\right)$ в классе $B_{1}^{l}$ хуже всего приближается функция

$$
\psi_{l}(t)=\left(t_{+}^{l-1}\right) /(l-1) !, \quad t_{+}=\max (0, t) ;
$$

точнее, имеет место такое утверждение.

Tеорема 7.3. Если $l=n-k, q=\infty, q^{\prime}=1,1 \leqslant p, r \leqslant \infty, 1 / p+1 / p^{\prime}=1$, $1 / r+1 / r^{\prime}=1$, то при любом $N>0$ справедливы равенства

$$
G(N)=F(N)=\inf \left\{\left\|\psi_{n-k}-\varphi\right\|_{L_{p^{\prime}}}: \varphi \in B_{r^{\prime}}^{n}(N)\right\}
$$

Подобные результаты для периодических функций были получены Б.Е. Клоцем [119], [133].

\section{СПИСОК ЛИТЕРАТУРЫ}

[1] Стечкин С. Б. Неравенства между нормами производных произвольной функции // Acta Sci. Math. 1965. T. 26. № 3-4. C. 225-230.

[2] Стечкин С. Б. Наилучшее приближение линейных операторов // Матем. заметки. 1967. T. 1. № 2. C. 137-148.

[3] Арестов В.В.О равномерной регуляризации задачи вычисления значений оператора // Матем. заметки. 1977. Т. 22. № 2. С. 231-244.

[4] Габушин В.Н. Оптимальные методы вычисления значений оператора $U x$, если $x$ задано с погрешностью. Дифференцирование функций, определенных с ошибкой // Тр. МИАН CCCP. 1980. T. 145 . C. $63-78$.

[5] Арестов В.В. Наилучшее восстановление операторов и родственные задачи // Тр. МИАН СССР. 1989. Т. 189. С. 3-20.

[6] Арестов В.В. Наилучшее приближение неограниченных операторов, инвариантных относительно сдвига, линейными ограниченными операторами // Тр. МИАН СССР. 1992. T. 198. C. $3-20$.

[7] Арестов В. В., Габушин В. Н. Наилучшее приближение неограниченных операторов ограниченными // Изв. ВУЗов. Матем. 1995. №11. С. 44-66.

[8] Иванов В. К., Королюк Т. И. Об оценке погрешности при решении линейных некорректных задач // Журн. вычисл. матем. и матем. физики. 1969. Т. 9. № 1. С. 30-41.

[9] Иванов В. К. Об оценке погрешности при решении операторных уравнений первого рода // Вопросы точности и эффект. вычисл. алгоритмов. Т. 2. Киев: Изд-во АН УССР, 1969. C. $102-116$. 
[10] Габушин В.Н. Наилучшее приближение функционалов на некоторых множествах // Матем. заметки. 1970. Т. 8. №5. С. 551-562.

[11] Лаврентьев М. М. О некоторых некорректных задачах математической физики. Новосибирск: Изд-во АН СССР, 1962.

[12] Тихонов А.Н., Арсенин В.Я. Методы решения некорректных задач. М.: Наука, 1974.

[13] Морозов В.А. Регулярные методы решения некорректно поставленных задач. М.: Изд-во МГУ, 1974.

[14] Стечкин С. Б., Субботин Ю. Н. Сплайныв вычислительной математике. М.: Наука, 1976.

[15] Иванов В. К., Васин В. В., Танана В. П. Теория линейных некорректных задач и ее приложения. М.: Наука, 1978.

[16] Лаврентьев М. М., Романов В. Г., Шишатский С. П. Некорректные задачи математической физики и анализа. М.: Наука, 1980.

[17] Танана В. П. Методы решения операторных уравнений. М.: Наука, 1981.

[18] Трауб Дж.Ф., Вожняковский Х. Общая теория оптимальных алгоритмов. М.: Мир, 1983.

[19] Гребенников А. И. Метод сплайнов и решение некорректных задач теории приближений. М.: Изд-во МГУ, 1983.

[20] Морозов В.А., Гребенников А. И. Методы решения некорректно поставленных задач. М.: Изд-во МГУ, 1992.

[21] Арсенин В.Я., Иванов В.В. Об оптимальной регуляризации // Докл. АН СССР. 1968. Т. 182. № 1. С. 9-12.

[22] Страхов В.Н. О решении линейных некорректных задач в гильбертовом пространстве // Дифференц. уравнения. 1970. Т. 6. №8. С. 1490-1495.

[23] Иванов В. В. Об оптимальных по точности алгоритмах приближенного решения операторных уравнений 1-го рода // Журн. вычисл. матем. и матем. физики. 1975. Т. 15. № 1. C. $3-11$.

[24] Марчук А. Г., Осипенко К. Ю. Наилучшее приближение функций, заданных с погрешностью в конечном числе точек // Матем. заметки. 1975. Т. 17. № 3. С. 359-368.

[25] Марчук А. Г. Оптимальные по точности методы решений линейных задач восстановления // Препринт ВЦ СО АН СССР. Новосибирск, 1976.

[26] Гребенников А.И., Морозов В.А. Об оптимальном приближении операторов // Журн. выгисл. матем. и матем. физики. 1977. Т. 17. №1. С. 3-14.

[27] Васин В.В.Оптимальные методы вычисления значений неограниченных операторов // Препринт АН УССР ИК. Киев, 1977. С. 77-59.

[28] Micchelli Ch. A., Rivlin Th. J. A survey of optimal recovery // Optimal estimation in approximation theory. New York: Plenum Press, 1977. P. 1-54.

[29] Bojanov B. Comparison theorems in optimal recovery // Optimal Algorithms. Sofia: BAN, 1986. P. 15-50.

[30] Женсыкбаев А. А. Сплайн-аппроксимация и оптимальное восстановление операторов // Матем. сб. 1993. Т. 184. № 12. С. 3-22.

[31] Zhensykbaev A. A. Recovery of operators on classes of multivariate functions // East J. Approximations. 1995. V. 1. № 2. P. 197-220.

[32] Танана В. П. Об оптимальности нелинейных методов при решении линейных неустойчивых задач // Оптимизация вычислительных методов. Киев: Изд-во ИК АН УССР, 1974. C. $52-58$.

[33] Peetre J. Approximation of linear operators // Tp. Междунар. конф. по конструктивной теории функций. Варна, 1970. София, 1972. С. 245-263.

[34] Габушин В.Н. Оценка экстремумов функции и ее производных // Поиск экстремума (матем. методы и автомат. системы). Тр. III Всесоюз. симпозиума по экстремальным задачам. Томск, 20-25 февр. 1967. Томск: Изд-во Томского ун-та, 1969. С. 243-244. 
[35] Бердышев В. И. Связь между неравенством Джексона и одной геометрической задачей // Матем. заметки. 1968. Т. 3. № 3. С. 327-338.

[36] Пичугов С. А. Константа Юнга пространств $L_{p} / /$ Матем. заметки. 1988. Т. 43. № 5. C. $604-614$

[37] Golomb M. Lectures on theory of approximation. Argonn Nat. Lab., Appl. Math. Division, 1962 .

[38] Мильман В. Д. Геометрическая теория пространств Банаха. Ч. II // УМН. 1971. Т. 26. №6. С. $73-149$.

[39] Корнейчук Н. П. Сплайны в теории приближения. М.: Наука, 1984.

[40] Смоляк С.А. Об оптимальном восстановлении функций и функционалов от них // Дис.... канд. физ.-матем. наук. М.: МГУ, 1965.

[41] Бахвалов Н. С. Об оптимальности линейных методов приближения операторов на выпуклых классах функций // Журн. вычисл. матем. и матем. физики. 1971. Т. 11.№ 4. C. $1014-1016$.

[42] Магарил-Ильяев Г. Г., Чан Тхи Ле. К задаче оптимального восстановления функционалов // УМН. 1987. Т. 42. №2. С. 237-238.

[43] Сухарев А. Г. Минимаксные алгоритмы в задачах численного анализа. М.: Наука, 1989.

[44] Магарил-Ильяев Г. Г., Осипенко К. Ю. Об оптимальном восстановлении функционалов по неточным данным // Матем. заметки. 1991. Т. 50. № 6. С. 85-93.

[45] Hardy G. H., Littlewood J. E. Contribution to the arithmetic theory of series // Proc. London Math. Soc. (2). 1912. V. 11. P. 411-478.

[46] Landau E. Einige Ungleichungen für zweimal differentierbare Funktionen // Proc. London Math. Soc. (2). 1913. V. 13. P. 43-49.

[47] Hadamard J. Sur le module maximum d'une fonction et de ses dérivées // Soc. Math. France, Comptes rendus des Séances. 1914. V. 41. P. 68-72.

[48] Колмогоров А. Н. О неравенствах между верхними гранями последовательных производных произвольной функции на бесконечном интервале // Избранные труды. Математика, механика. М.: Наука, 1985. С. 252-263.

[49] Sz.-Nagy B. Über Integralungleichungen zwischen einer Function und ihrer Ableitung // Acta Sci. Math. 1941. V. 10. P. 64-74.

[50] Габушин В.Н. Неравенства между производными в метриках $L_{p}$ при $0<p \leqslant \infty / /$ Изв. АН СССР. Сер. матем. 1976. Т. 40. № 40. С. 869-892.

[51] Габушин В.Н. Неравенства для норм функции и ее производных в метриках $L_{p} / /$ Матем. заметки. 1967. Т. 1. №3. С. 291-298.

[52] Габушин В.Н.Неравенства для производных решений обыкновенных дифференциальных уравнений в метриках $L_{p}(0<p \leqslant \infty) / /$ Дифференц. уравнения. 1988. Т. 24. № 10 . C. $1662-1670$.

[53] Боссе Ю. Г. (Шилов Г. Е.). О неравенствах между производными // Сб. работ студ. научн. кружков МГУ. Т. 1, 1937. С. 68-72.

[54] Харди Г., Литтлвуд Дж., Полиа Г. Неравенства. М.: ИЛ, 1948.

[55] Stein E. M. Functions of exponential type // Ann. of Math. (2). 1957. V. 65. P. 582-592.

[56] Тайков Л.В.Неравенства типа Колмогорова и наилучшие формулы численного дифференцирования // Матем. заметки. 1968. Т. 4. № 2. С. 233-238.

[57] Габушин В. Н. Точные константы в неравенствах между нормами производных функций // Матем. заметки. 1968. Т. 4. №2. С. 221-232.

[58] Габушин В.Н. Некоторые неравенства между производными функций // Тр. Ин-та математики и механики УНЦ АН СССР. 1976. № 23. Методы регуляризации неустойчивых задач. С. 20-26.

[59] Буслаев А.П. О точных константах и неравенствах для производных // Школа по теории операторов в функциональных пространствах. Тез. докл. Минск, 1982. С. 29.

[60] Арестов В.В. О точных неравенствах между нормами функций и их производных // Acta Sci. Math. 1972. T. 33. № 3-4. C. 243-267. 
[61] Магарил-Ильяев Г. Г. Вложение обобщенных соболевских классов и неравенства для производных // Дис. ... канд. физ.-матем. наук. М., 1980.

[62] Магарил-Ильяев Г. Г. Неравенства для производных и двойственность // Тр. МИАН CCCP. 1983. T. 161. C. $183-194$.

[63] Соляр В.Г. Об одном неравенстве между нормами функции и ее производных // Изв. ВУЗов. Матем. 1976. № 2. С. 165-168.

[64] Арестов В.В., Бердышев В.И. Неравенства для дифференцируемых функций // Тр. Ин-та математики и механики УНЦ АН СССР. 1975.№17. Методы решения условно-корректных задач. С. 108-138.

[65] Маторин А. П. О неравенствах между наибольшими значениями абсолютных величин функции и ее производных на полупрямой // Укр. матем. журн. 1955. Т. 7. С. 262-266.

[66] Габушин В.Н. О наилучшем приближении оператора дифференцирования на полупрямой // Матем. заметки. 1969. Т. 6. № 5. С. 573-582.

[67] Купцов Н.П. О точных константах в неравенствах между нормами функций и их производных // Матем. заметки. 1987. Т. 41. № 3. С. 313-319.

[68] Бердышев В. И. Наилучшее приближение в $L[0, \infty)$ оператора диффференцирования // Матем. заметки. 1971. Т. 9. №5. С. 477-481.

[69] Магарил-Ильяев Г. Г. О неравенствах Колмогорова на полупрямой // Вестник МГУ. Сер. матем., мех. 1976. № 5. С. 33-41.

[70] Любич Ю.И. О неравенствах между степенями линейного оператора // Изв. АН CССР. Сер. матем. 1960. Т. 24. С. 825-864.

[71] Купцов Н. П. Колмогоровские оценки для производных в $L_{2}[0, \infty) / /$ Тр. МИАН СССР. 1975. T. 138. C. $94-117$.

[72] Буслаев А. П., Магарил-Ильяев Г. Г., Тихомиров В. М. О существовании экстремальной функции в неравенстве для производных // Матем. заметки. 1982. Т. 32. №6. C. 823-834.

[73] Kwong M. K., Zettl A. Ramifications of Landau's inequality // Proc. Roy. Soc. Edinburgh. Sect. A. 1980. V. 86. P. 175-212.

[74] Schoenberg I. J., Cavaretta A. Solution of Landau's problem concerning higher derivatives on the halfline // Proc. Int. Conf. on Constructive Function Theory. Varna, May 19-25, 1970. Sofia, 1972. P. 297-308.

[75] Стечкин С.Б. О неравенствах между верхними гранями производных произвольной функции на полупрямой // Матем. заметки. 1967. Т. 1. № 6. С. 665-674.

[76] Kato T. On an Inequality of Hardy, Littlewood, and Pólya // Adv. in Math. 1971. V. 7. №3. P. 217-218.

[77] Буслаев А. П. Об одной экстремальной задаче, связанной с неравенствами для производных // Вестник МГУ. Сер. матем., мех. 1978. № 3. С. 67-78.

[78] Everitt W. D. On an extension to an integro-differential inequality of Hardy, Littlewood and Polya // Proc. Roy. Soc. Edinburgh. Sect. A. 1972. V. 69. P. 295-333.

[79] Everitt W. D., Giertz M. Some inequalities assotiated with certain ordinary differential operators // Math. Z. 1972. № 126. P. 308-326.

[80] Everitt W. D., Giertz M. On the integro-differential inequality $\left\|f^{\prime}\right\|_{2}^{2} \leqslant K\|f\|_{p}\left\|f^{\prime \prime}\right\|_{q}$ // J. Math. Anal. Appl. 1974. № 45. P. 639-653.

[81] Everitt W. D., Giertz M. Inequalities and separation for certain ordinary differential operators // Proc. London Math. Soc. 1974. V. 3. № 28. P. 352-372.

[82] Everitt W. D., Jones D. S. On an integral inequality // Proc. Roy. Soc. London. Sect. A. 1977. V. 357. P. 271-288.

[83] Everitt W. D., Zettl A. On a class of integral inequalities // J. London Math. Soc. (2). 1978. V. 17. P. 291-303.

[84] Kwong M. K., Zettl A. Norm Inequalities for Derivatives and Differences // Lect. Notes in Math. V. 1536. Berlin: Springer-Verlag, 1992.

[85] Тихомиров В.М., Магарил-Ильяев Г. Г. Неравенства для производных // Колмогоров А.Н. Избранные труды. Математика и механика. М.: Наука, 1985. С. 387-390. 
[86] Арестов В.В. Наилучшие приближения операторов дифференцирования // Тез. крат. науч. сообщ. на Междунар. конгр. математиков. Москва, 1966. Секция 4. М., 1966. C. $31-32$.

[87] Арестов В. В. О наилучшем приближении операторов дифференцирования // Матем. заметки. 1967. Т. 1. № 2. С. 149-154.

[88] Domar Y. An extremal problem related to Kolmogoroff's inequality for bounded functions // Ark. Mat. 1968. V. 7. P. 433-441.

[89] Буслаев А.П. О приближении оператора дифференцирования // Матем. заметки. 1981. T. 25. № 5. С. 731-742.

[90] Субботин Ю. Н., Тайков Л.В.Наилучшее приближение оператора дифференцирования в пространстве $L_{2} / /$ Матем. заметки. 1968. Т. 3. № 2. С. 257-264.

[91] Арестов В.В. Приближение операторов, инвариантных относительно сдвига // Тр. МИАН СССР. 1975. Т. 138. С. 43-70.

[92] Арестов В. В. О наилучшем приближении операторов дифференцирования в равномерной метрике // Дис. . . . канд. физ.-матем. наук. Свердловск, 1969.

[93] Буслаев А.П. Приближение оператора диффференцирования и неравенства для производных // Дис. ... канд. физ.-матем. наук. М.: МГУ, 1979.

[94] Арестов В.В.О наилучшем равномерном приближении операторов дифференцирования // Матем. заметки. 1969. Т. 5. № 3. С. 273-284.

[95] Бесов О. В., Ильин В. П., Никольский С. М. Интегральные представления функций и теоремы вложения. М.: Наука, 1975.

[96] Габушин В. Н. О наилучшем приближении оператора дифференцирования в метрике $L_{p}$ // Матем. заметки. 1972. Т. 12. №5. С. 531-538.

[97] Габушин В.Н. Наилучшие приближения операторов // Теория приближения функций. Тр. Междунар. конф. Теория приближения функций. Калуга, 24-28 июля 1975 г. М.: Наука, 1977. С. $97-100$.

[98] Габушин В.Н. К задаче о наилучшем приближении операторов // Методы решения условно корректных задач. Тр. Ин-та математики и механики УНЦ АН СССР. № 17. Свердловск, 1975. С. $90-107$.

[99] Арестов В. В. Приближение инвариантных операторов // Матем. заметки. 1983. Т. 34. № 1. C. $9-29$.

[100] Berdysheva E. E. On the best approximation of the differentiation operator in $L_{2}(0, \infty)$ // East J. Approximations. 1996. V. 2. № 3. P. 315-321.

[101] Berdyshev S. V. Approximation of differentiation operators on classes of twice differentiable functions on the half-line // East J. Approximations. 1996. V. 2. № 1. P. 49-69.

[102] Arestov V. V. Inequalities for fractional derivatives on the half-line // Approx. Theory. Banach Center Publ. Warsaw. 1979. V. 4. P. 19-34.

[103] Коновалов В. Н. Точные неравенства для норм функций, третьих частных, вторых смешанных или косых производных // Матем. заметки. 1978. Т. 23. № 1. С. 67-78.

[104] Тимошин О.А. Наилучшее приближение оператора второй смешанной производной в метриках $L$ и $C$ на плоскости // Матем. заметки. 1984. Т. 36. № 3. С. 369-375.

[105] Тимошин О.А. Точные неравенства между нормами частных производных второго и третьего порядка // ДАН. 1995. Т. 344. №1. С. 20-22.

[106] Тимофеев В. Г. Неравенство типа Ландау для функций нескольких переменных // Матем. заметки. 1985. Т. 37. № 5. С. 676-689.

[107] Gagliardo E. Proprieta di alcune classi di funzion in piu variabili // Ricerche Math. 1958. V. 8. №7. P. 102-137.

[108] Nirenberg L. On elliptic partial differential equations // Ann. Scuola Norm. Super. Pisa. 1959. V. 13. P. $115-162$.

[109] Бесов О.В.Мультипликативные оценки для интегральных норм дифференцируемых функций многих переменных // Тр. МИАН СССР. 1974. Т. 131. С. 3-15.

[110] Peetre J. A remark on Sobolev spaces. The case $0<p<1 / /$ J. Approx. Theory. 1975. V. 13. № 3. P. 218-238. 
[111] Буслаев А. П., Тихомиров В. М. О неравенствах для производных в многомерном случае // Матем. заметки. 1979. Т. 25. №1. С. 59-74.

[112] Габушин В.Н. Неравенства между производными в метриках $L_{p}(0<p \leqslant \infty)$ для функций многих переменных. Свердловск: Ин-т математики и механики УНЦ АН СССР. 1984. Рукопись деп. в ВИНИТИ 27.03.84, № 1665-84 Деп.

[113] Габушин В.Н. Неравенства между производными в метриках $L_{p}$ при $0<p \leqslant \infty$ для функций многих переменных и оптимальное дифференцирование функций // Науч. докл. УрО РАН. Свердловск: Ин-т математики и механики, 1988.

[114] Габушин В.Н.О дифференцировании функций, определенных с ошибкой // Всес. конф. по некорректно-поставленным задачам. Фрунзе, 1979. Тез. докл. Фрунзе: ИЛИМ, 1979. C. 37 .

[115] Габушин В.Н. Оценки модулей непрерывности операторов // Алгоритмический и численньй анализ некорректных задач. Екатеринбург, 1995. С. 45-46.

[116] Арестов В. В.Приближение операторов типа свертки линейными ограниченными операторами // Тр. МИАН СССР. 1980. Т. 145. С. 3-19.

[117] Lorentz G. G. Approximation of function. New York: Holt, Renehat and Winston, 1966.

[118] Тихомиров В. М. Некоторые вопросы теории приближений. М.: Изд-во МГУ, 1976.

[119] Клоц Б. Е. Линейные уклонения классов $\widetilde{W}_{p}^{\alpha}$ и приближения в пространствах мультипликаторов // Матем. заметки. 1975. Т. 18. №1. С. 97-108.

[120] Гринлиф $\Phi$. Инвариантные средние на топологических группах и их приложения. М.: Мир, 1973.

[121] Хьюитт Э., Росс К. Абстрактный гармонический анализ. М.: Наука, 1975.

[122] Хермандер Л. Оценки для операторов, инвариантных относительно сдвига. М.: ИЛ, 1962.

[123] Larsen R. An introduction to the theory of multipliers. Berlin: Springer-Verlag, 1971.

[124] Стейн И., Вейс Г. Введение в гармонический анализ на евклидовых пространствах. М.: Мир, 1974.

[125] Арестов В.В.О наилучшем приближении оператора дифференцирования // Приближение функций полиномами и сплайнами. Свердловск: УНЦ АН СССР, 1985. С. 3-14.

[126] Арестов В.В.Приближение линейных операторов и родственные экстремальые задачи // Тр. МИАН СССР. 1975. Т. 138. С. 29-42.

[127] Арестов В.В.О некоторых экстремальных задачах для дифференцируемых функций одной переменной // Тр. МИАН СССР. 1975. Т. 138. С. 3-28.

[128] Корнейчук Н. П. Экстремальные задачи теории приближения. М.: Наука, 1976.

[129] Арестов В.В., Габушин В.Н.О приближении классов дифференцируемых функций // Матем. заметки. 1971. Т. 9. № 2. С. 105-112.

[130] Субботин Ю.Н. Связь сплайн приближений с задачей приближения класса классом // Матем. заметки. 1971. Т. 9. №5. С. 501-510.

[131] Субботин Ю. Н. Наилучшее приближение класса функций другим классом // Матем. заметки. 1967. Т. 2. № 5. С. 495-504.

[132] Тайков Л. В. О наилучшем приближении в среднем некоторых классов аналитических функций // Матем. заметки. 1967. Т. 1. № 2. С. 155-162.

[133] Клоц Б. Е. Приближения дифференцируемых функций функциями большей гладкости // Матем. заметки. 1977. Т. 21. № 1. С. 21-32. 